\title{
Rotational hypersurfaces in Lorentz-Minkowski 4-space
}

\author{
Mustafa Altin*1 (D), Ahmet Kazan² (D) \\ ${ }^{1}$ Technical Sciences Vocational School, Bingöl University, Bingöl, Turkey \\ ${ }^{2}$ Department of Computer Technologies, Doğanşehir Vahap Küçük Vocational School of Higher Education, \\ Malatya Turgut Özal University, Malatya, Turkey
}

\begin{abstract}
In this study, we study rotational hypersurfaces in 4-dimensional Lorentz-Minkowski space. We find the rotational hypersurfaces about spacelike axis according to Gaussian and mean curvatures in $E_{1}^{4}$ and give some results with the aid of the Gaussian and mean curvatures. After that, we deal with the Gauss map of rotational hypersurface about spacelike axis by obtaining the Gaussian and mean curvatures. We obtain the second and third LaplaceBeltrami operators on rotational hypersurface about spacelike axis in $E_{1}^{4}$. Also, we give these characterizations for rotational hypersurfaces about timelike and lightlike axes, too.
\end{abstract}

Mathematics Subject Classification (2020). 14J70, 53A35

Keywords. rotational hypersurface, Gauss map, second Laplace-Beltrami operator, third Laplace-Beltrami operator

\section{Introduction}

It is known that a rotational hypersurface is defined as a hypersurface rotating a curve around an axis. In this context, if $\alpha: I \subset R \longrightarrow \pi$ is a curve in a plane $\pi$ in 4dimensional Lorentz-Minkowski space $E_{1}^{4}$ and $l$ is a straight line in $E_{1}^{4}$, then a rotational hypersurface is defined by a hypersurface rotating the profile curve $\alpha$ around the axis $l$. Furthermore, if the profile curve $\alpha$ rotates around the axis $l$ and it simultaneously displaces parallel lines orthogonal to the axis $l$, then the obtained hypersurface is called helicoidal hypersurface with the axis $l$. With the aid of these definitions, the differential geometry of rotational (hyper)surfaces, helicoidal (hyper)surfaces or other types of (hyper)surfaces in 3 or higher-dimensional Euclidean, Minkowskian, Galilean, and pseudo-Galilean spaces have been studied by scientists. For instance, finite type surfaces of revolution in a Euclidean 3 -space have been classified in [6] and some properties about surfaces of revolution in four dimensions have been given in [17]. In [5], the authors have studied the translation surfaces in the 3-dimensional Euclidean and Lorentz-Minkowski spaces under the condition $\Delta^{I I I} r_{i}=\mu_{i} r_{i}, \mu_{i} \in \mathbb{R}$, where $\Delta^{I I I}$ denotes the Laplacian of the surface with respect to the nondegenerate third fundamental form $I I I$ and in [8], the authors have classified the translation surfaces in three dimensional Galilean space $G^{3}$ satisfying $\Delta^{I I} x_{i}=\lambda_{i} x_{i}$,

\footnotetext{
*Corresponding Author.

Email addresses: maltin@bingol.edu.tr (M. Altın), ahmet.kazan@ozal.edu.tr (A. Kazan)

Received: 16.11.2020; Accepted: 03.05.2021
} 
$\lambda_{i} \in \mathbb{R}$, where $\Delta^{I I}$ denotes the Laplacian of the surface with respect to the nondegenerate second fundamental form $I I$ (throughout this study, we call the operators $\Delta^{I I}$ and $\Delta^{I I I}$ as second Laplace-Beltrami operator and third Laplace-Beltrami operator, respectively). The general rotational surfaces in Minkowski 4-space and the third Laplace-Beltrami operator and the Gauss map of the rotational hypersurface in Euclidean 4-space have been studied in [10] and [14], respectively. Also, Dini-type helicoidal hypersurface in $E^{4}$ and Dinitype helicoidal hypersurfaces with timelike axis in $E_{1}^{4}$ have been studied in [12] and [13], respectively. In [7], the authors have been classified complete hypersurfaces in $E^{4}$ with constant mean curvature and constant scalar curvature. In [2], Arslan and his friends have considered generalized rotational surfaces imbedded in a Euclidean space of four dimensions and also they have given some special examples of these surfaces in $E^{4}$ and in [3], the authors have studied translation surfaces in Euclidean 4-space. Hypersurfaces in Euclidean 4-space with harmonic mean curvature vector field have been studied in [15]. In [19], Yoon has studied rotational surfaces with finite type Gauss map in Euclidean 4-space. Minimal translation hypersurfaces in $E^{4}$ have been studied by Moruz and Munteanu [18]. Also, in [1], the authors have studied the Monge hypersurfaces in Euclidean 4-space with density. Furthermore, Izumiya et al. have introduced the notion of flatness for lightlike hypersurfaces and studied their singularities [16]. In [4], the authors have studied Lorentz hypersurfaces in $E_{1}^{4}$ satisfying $\Delta \vec{H}=\alpha \vec{H}$, where $\vec{H}$ is the mean curvature vector field of a hypersurface, $\Delta$ is Laplace operator and $\alpha$ is a constant and they have shown that the Lorentz hypersurface satisfying this condition has constant mean curvature. The explicit parameterizations of rotational hypersurfaces in Lorentz-Minkowski space $E_{1}^{n}$ have been given and rotational hypersurfaces in $E_{1}^{n}$ with constant mean curvature have been obtained in [9]. In [11], the author has found the equations for Gaussian and mean curvatures of the helicoidal hypersurfaces in $E_{1}^{4}$. Also, he has obtained a theorem classifying the helicoidal hypersurface with timelike axis satisfying $\Delta H=A H$, where $A$ is a $4 \times 4$ matrix.

In the present paper, we study the rotational hypersurfaces in 4-dimensional LorentzMinkowski space. In this context, firstly we give the Gaussian and mean curvatures of rotational hypersurfaces (which are special types of helicoidal hypersurfaces studied in [11]) about spacelike, timelike and lightlike axes in $E_{1}^{4}$. Also, we find the rotational hypersurfaces about spacelike and timelike axes according to the Gaussian and mean curvatures in $E_{1}^{4}$ and give some results with the aid of these curvatures. After that, we deal with the Gauss map of rotational hypersurfaces about spacelike, timelike and lightlike axes by obtaining the Gaussian and mean curvatures. Also, we study the second and third Laplace-Beltrami ( $\mathrm{LB}^{\mathrm{II}}$ and $\mathrm{LB}^{\mathrm{III}}$ ) operators on rotational hypersurface about spacelike, timelike and lightlike axes in $E_{1}^{4}$.

Now, let us recall some fundamental notions for hypersurfaces in Lorentz-Minkowski 4-space.

If $\vec{x}=\left(x_{1}, x_{2}, x_{3}, x_{4}\right), \vec{y}=\left(y_{1}, y_{2}, y_{3}, y_{4}\right)$ and $\vec{z}=\left(z_{1}, z_{2}, z_{3}, z_{4}\right)$ are three vectors in $E_{1}^{4}$, then the inner product and vector product are defined by

$$
\langle\vec{x}, \vec{y}\rangle=-x_{1} y_{1}+x_{2} y_{2}+x_{3} y_{3}+x_{4} y_{4}
$$

and

$$
\vec{x} \times \vec{y} \times \vec{z}=\operatorname{det}\left[\begin{array}{cccc}
-e_{1} & e_{2} & e_{3} & e_{4} \\
x_{1} & x_{2} & x_{3} & x_{4} \\
y_{1} & y_{2} & y_{3} & y_{4} \\
z_{1} & z_{2} & z_{3} & z_{4}
\end{array}\right]
$$

respectively. Also, the norm of the vector $\vec{x}$ is $\|\vec{x}\|=\sqrt{|\langle\vec{x}, \vec{x}\rangle|}$. 
If

$$
\begin{aligned}
\Gamma: U \subset E^{3} & \longrightarrow E_{1}^{4} \\
\left(u_{1}, u_{2}, u_{3}\right) & \longrightarrow \Gamma\left(u_{1}, u_{2}, u_{3}\right)=\left(\Gamma_{1}\left(u_{1}, u_{2}, u_{3}\right), \Gamma_{2}\left(u_{1}, u_{2}, u_{3}\right), \Gamma_{3}\left(u_{1}, u_{2}, u_{3}\right), \Gamma_{4}\left(u_{1}, u_{2}, u_{3}\right)\right)
\end{aligned}
$$

is a hypersurface in $E_{1}^{4}$, then the Gauss map (i.e., the unit normal vector field), the matrix forms of the first and second fundamental forms are

$$
\begin{gathered}
N_{\Gamma}=\frac{\Gamma_{u_{1}} \times \Gamma_{u_{2}} \times \Gamma_{u_{3}}}{\left\|\Gamma_{u_{1}} \times \Gamma_{u_{2}} \times \Gamma_{u_{3}}\right\|}, \\
{\left[g_{i j}\right]=\left[\begin{array}{lll}
g_{11} & g_{12} & g_{13} \\
g_{21} & g_{22} & g_{23} \\
g_{31} & g_{32} & g_{33}
\end{array}\right]}
\end{gathered}
$$

and

$$
\left[h_{i j}\right]=\left[\begin{array}{lll}
h_{11} & h_{12} & h_{13} \\
h_{21} & h_{22} & h_{23} \\
h_{31} & h_{32} & h_{33}
\end{array}\right]
$$

respectively. Here $g_{i j}=\left\langle\Gamma_{u_{i}}, \Gamma_{u_{j}}\right\rangle, h_{i j}=\left\langle\Gamma_{u_{i} u_{j}}, N_{\Gamma}\right\rangle, \Gamma_{u_{i}}=\frac{\partial \Gamma}{\partial u_{i}}, \Gamma_{u_{i} u_{j}}=\frac{\partial^{2} \Gamma}{\partial u_{i} u_{j}}, i, j \in$ $\{1,2,3\}$.

Also, the matrix of shape operator of the hypersurface (1.3) is

$$
S=\left[a_{i j}\right]=\left[g^{i j}\right] \cdot\left[h_{i j}\right]
$$

where $\left[g^{i j}\right]$ is the inverse matrix of $\left[g_{i j}\right]$.

With the aid of (1.5)-(1.7), the Gaussian curvature and mean curvature of a hypersurface in $E_{1}^{4}$ are given by

$$
K=\varepsilon \frac{\operatorname{det}\left[h_{i j}\right]}{\operatorname{det}\left[g_{i j}\right]}
$$

and

$$
3 \varepsilon H=\operatorname{tr}(S),
$$

respectively. Here, $\varepsilon=\left\langle N_{\Gamma}, N_{\Gamma}\right\rangle$. For more details about hypersurfaces in $E_{1}^{4}$, we refer to $[11,13]$ and etc. Also, the inverse of an arbitrary matrix

$$
\left[A_{i j}\right]=\left[\begin{array}{lll}
A_{11} & A_{12} & A_{13} \\
A_{21} & A_{22} & A_{23} \\
A_{31} & A_{32} & A_{33}
\end{array}\right]
$$

in $E_{1}^{4}$ is

$$
\left[A^{i j}\right]=\frac{1}{\operatorname{det}\left[A_{i j}\right]}\left[\begin{array}{ccc}
A_{22} A_{33}-A_{23} A_{32} & A_{13} A_{32}-A_{12} A_{33} & A_{12} A_{23}-A_{13} A_{22} \\
A_{23} A_{31}-A_{21} A_{33} & A_{11} A_{33}-A_{13} A_{31} & A_{13} A_{21}-A_{11} A_{23} \\
A_{21} A_{32}-A_{22} A_{31} & A_{12} A_{31}-A_{11} A_{32} & A_{11} A_{22}-A_{12} A_{21}
\end{array}\right]
$$

where

$\operatorname{det}\left[A_{i j}\right]=-A_{13} A_{22} A_{31}+A_{12} A_{23} A_{31}+A_{13} A_{21} A_{32}-A_{11} A_{23} A_{32}-A_{12} A_{21} A_{33}+A_{11} A_{22} A_{33}$.

In the present study, we deal with timelike rotational hypersurfaces. One can obtain corresponding results with same methods for spacelike rotational hypersurfaces, too. 


\section{Rotational hypersurfaces about spacelike axis in $E_{1}^{4}$}

In this section, we find the rotational hypersurface about spacelike axis according to the Gaussian and mean curvatures in $E_{1}^{4}$ and give some examples for different Gaussian and mean curvatures. We study the Gauss map of this hypersurface and obtain the curvatures of it. Also, we study the $\mathrm{LB}^{\mathrm{II}}$ and $\mathrm{LB}^{\mathrm{III}}$ operators on the rotational hypersurface with spacelike axis in $E_{1}^{4}$ and give some characterizations for $\mathrm{LB}^{\mathrm{II}}$-minimality and $\mathrm{LB}^{\mathrm{III}}$ minimality of this hypersurface.

\subsection{Curvatures of rotational hypersurfaces about spacelike axis in $E_{1}^{4}$}

For a differentiable function $f(x): I \subset \mathbb{R} \longrightarrow \mathbb{R}$, the rotational hypersurface which is obtained by rotating the profile curve $\alpha(x)=(x, 0,0, f(x))$ about spacelike axis $(0,0,0,1)$ is given by

$$
\begin{aligned}
\Gamma(x, y, z) & =\left[\begin{array}{cccc}
\cosh y \cosh z & \sinh y \cosh z & \sinh z & 0 \\
\sinh y & \cosh y & 0 & 0 \\
\cosh y \sinh z & \sinh y \sinh z & \cosh z & 0 \\
0 & 0 & 0 & 1
\end{array}\right] \cdot\left[\begin{array}{c}
x \\
0 \\
0 \\
f(x)
\end{array}\right] \\
& =(x \cosh y \cosh z, x \sinh y, x \cosh y \sinh z, f(x)),
\end{aligned}
$$

where $x \in \mathbb{R}-\{0\}$.

With the aid of the first differentials of (2.1) with respect to $x, y$ and $z$, the Gauss map of the rotational hypersurface (2.1) is obtained from (1.4) by

$$
N_{\Gamma}=-\frac{1}{\sqrt{1-f^{\prime 2}}}\left(f^{\prime} \cosh y \cosh z, f^{\prime} \sinh y, f^{\prime} \cosh y \sinh z, 1\right)
$$

and from (2.2), we get

$$
\left\langle N_{\Gamma}, N_{\Gamma}\right\rangle=1 .
$$

Here, we state $f=f(x)$ and $f^{\prime}=\frac{d f(x)}{d x}$.

Also, from (1.5), we obtain the matrix of the first fundamental form, its inverse and determinant as

$$
\begin{gathered}
{\left[g_{i j}\right]=\left[\begin{array}{ccc}
f^{\prime 2}-1 & 0 & 0 \\
0 & x^{2} & 0 \\
0 & 0 & x^{2} \cosh ^{2} y
\end{array}\right]} \\
{\left[g^{i j}\right]=\left[\begin{array}{ccc}
\frac{1}{f^{\prime 2}-1} & 0 & 0 \\
0 & \frac{1}{x^{2}} & 0 \\
0 & 0 & \frac{1}{x^{2} \cosh ^{2} y}
\end{array}\right]}
\end{gathered}
$$

and

$$
\operatorname{det}\left[g_{i j}\right]=x^{4}\left(f^{\prime 2}-1\right) \cosh ^{2} y,
$$

respectively. From (1.6), the matrix form of the second fundamental form of the hypersurface (2.1), its inverse and determinant as

$$
\begin{gathered}
{\left[h_{i j}\right]=\frac{1}{\sqrt{1-f^{\prime 2}}}\left[\begin{array}{ccc}
-f^{\prime \prime} & 0 & 0 \\
0 & x f^{\prime} & 0 \\
0 & 0 & x f^{\prime} \cosh ^{2} y
\end{array}\right],} \\
{\left[h^{i j}\right]=\sqrt{1-f^{\prime 2}}\left[\begin{array}{ccc}
-\frac{1}{f^{\prime \prime}} & 0 & 0 \\
0 & \frac{1}{x f^{\prime}} & 0 \\
0 & 0 & \frac{1}{x f^{\prime} \cosh ^{2} y}
\end{array}\right]}
\end{gathered}
$$

and

$$
\operatorname{det}\left[h_{i j}\right]=-\frac{x^{2} f^{\prime 2} f^{\prime \prime} \cosh ^{2} y}{\left(1-f^{\prime 2}\right)^{3 / 2}}
$$


respectively and here, we state $f^{\prime \prime}=\frac{d^{2} f(x)}{d x^{2}}$. Hence, using (2.3), (2.6) and (2.9) in (1.8), we can give the following theorem:

Theorem 2.1. The Gaussian curvature of the rotational hypersurface (2.1) is

$$
K=\frac{f^{\prime 2} f^{\prime \prime}}{x^{2}\left(1-f^{\prime 2}\right)^{\frac{5}{2}}} .
$$

Here, we want to find the function $f$ according to the Gaussian curvature $K$ by solving the equation (2.10). For solving the differential equation (2.10), let us put

$$
A=\frac{f^{\prime 3}}{x^{6}\left(1-f^{\prime 2}\right)^{3 / 2}} .
$$

By differentiating (2.11) and using (2.10), we have

$$
A^{\prime}=\frac{3 K(x)-6 x^{3} A}{x^{4}} .
$$

The solution of (2.12) which is a first order differential equation with respect to $A$ is obtained by

$$
A=\frac{3 \int_{1}^{x} K(t) t^{2} d t+c_{1}}{x^{6}}
$$

$c_{1} \in \mathbb{R}$. From (2.11) and (2.13), we obtain that

$$
\left(1-f^{\prime 2}\right)^{3 / 2}\left(3 \int_{1}^{x} K(t) t^{2} d t+c_{1}\right)=f^{\prime 3}
$$

and so,

$$
f(x)= \pm \int \frac{\left(3 \int_{1}^{x} K(t) t^{2} d t+c_{1}\right)^{\frac{1}{3}}}{\sqrt{1+\left(3 \int_{1}^{x} K(t) t^{2} d t+c_{1}\right)^{\frac{2}{3}}}} d x .
$$

Hence, we can state the following theorem:

Theorem 2.2. The rotational hypersurface (2.1) about spacelike axis in $E_{1}^{4}$ can be parametrized with respect to the Gaussian curvature by

$\Gamma(x, y, z)=\left(x \cosh y \cosh z, x \sinh y, x \cosh y \sinh z, \pm \int \frac{\left(3 \int_{1}^{x} K(t) t^{2} d t+c_{1}\right)^{\frac{1}{3}}}{\sqrt{1+\left(3 \int_{1}^{x} K(t) t^{2} d t+c_{1}\right)^{\frac{2}{3}}}} d x\right)$

where $c_{1} \in \mathbb{R}$.

Example 2.3. If we take $K(x)=\frac{1}{x^{2}}$ and $c_{1}=3$ in (2.16), then the rotational hypersurface is

$$
\Gamma(x, y, z)=\left(x \cosh y \cosh z, x \sinh y, x \cosh y \sinh z, \frac{\left(\sqrt[3]{9 x^{2}}-2\right) \sqrt{1+\sqrt[3]{9 x^{2}}}}{3}\right) .
$$

In the following figures, one can see the projections of the rotational hypersurface (2.17) for $z=2$ into $x_{2} x_{3} x_{4}, x_{1} x_{3} x_{4}, x_{1} x_{2} x_{4}$ and $x_{1} x_{2} x_{3}$-spaces in (a), (b), (c) and (d), respectively. 


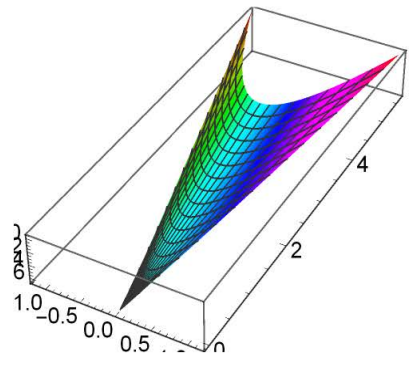

(a)

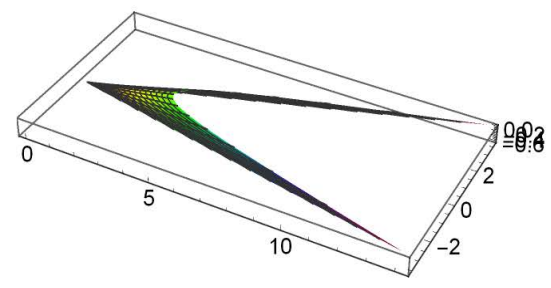

(c)

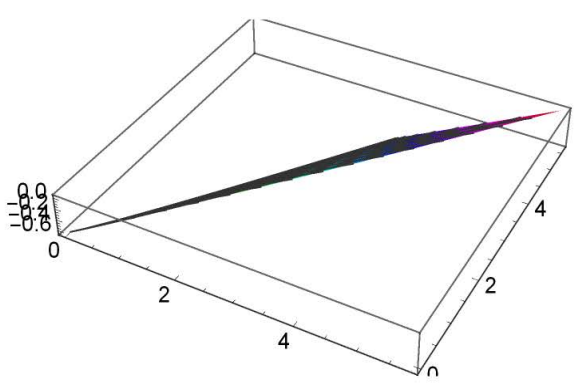

(b)

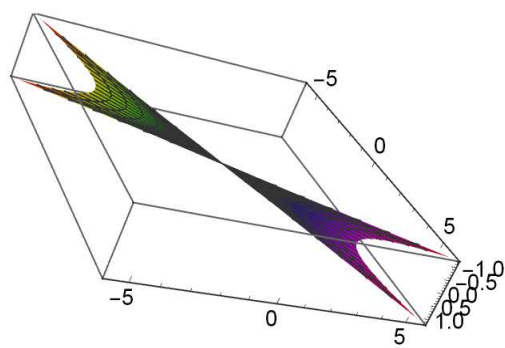

(d)

Figure 1

Also from Theorem 2.2, we can give the following results:

Corollary 2.4. The rotational hypersurface (2.1) about spacelike axis in $E_{1}^{4}$ with constant Gaussian curvature $(K=k \in \mathbb{R})$ can be parametrized by

$$
\Gamma(x, y, z)=\left(x \cosh y \cosh z, x \sinh y, x \cosh y \sinh z, \pm \int \frac{\sqrt[3]{c_{1}+k\left(x^{3}-1\right)}}{\sqrt{1+\sqrt[3]{\left(c_{1}+k\left(x^{3}-1\right)\right)^{2}}}} d x\right) .
$$

Corollary 2.5. The rotational hypersurface (2.1) about spacelike axis in $E_{1}^{4}$ with zero Gaussian curvature can be parametrized by

$$
\Gamma(x, y, z)=\left(x \cosh y \cosh z, x \sinh y, x \cosh y \sinh z, \pm \frac{\sqrt[3]{c_{1}} x}{\sqrt{1+\sqrt[3]{\left(c_{1}\right)^{2}}}}\right) .
$$

Also, using (2.5) and (2.7) in (1.7), the shape operator of the rotational hypersurface (2.1) is obtained by

$$
S=\frac{1}{\sqrt{1-f^{\prime 2}}}\left[\begin{array}{ccc}
\frac{f^{\prime \prime}}{1-f^{\prime 2}} & 0 & 0 \\
0 & \frac{f^{\prime}}{x} & 0 \\
0 & 0 & \frac{f^{\prime}}{x}
\end{array}\right]
$$

So, from (1.9), (2.3) and (2.18), we get

Theorem 2.6. The mean curvature of the rotational hypersurface (2.1) is

$$
H=\frac{2 f^{\prime}\left(1-f^{\prime 2}\right)+x f^{\prime \prime}}{3 x\left(1-f^{\prime 2}\right)^{3 / 2}} .
$$

Here, we want to find the function $f$ according to the mean curvature $H$ by solving the equation (2.19). For solving the differential equation (2.19), let us take

$$
B=\frac{f^{\prime}(x)}{x \sqrt{1-f^{\prime 2}(x)}} .
$$


By differentiating (2.20) and using (2.19), we have

$$
B^{\prime}=\frac{3 H(x)-3 B}{x} .
$$

The solution of (2.21) which is a first order differential equation with respect to $B$ is obtained by

$$
B=\frac{3 \int_{1}^{x} H(t) t^{2} d t+c_{2}}{x^{3}},
$$

$c_{2} \in \mathbb{R}$. From (2.20) and (2.22), we obtain that

$$
f(x)= \pm \int \frac{3 \int_{1}^{x} H(t) t^{2} d t+c_{2}}{\sqrt{x^{4}+\left(3 \int_{1}^{x} H(t) t^{2} d t+c_{2}\right)^{2}}} d x .
$$

Thus, we can give the following theorem:

Theorem 2.7. The rotational hypersurface (2.1) about spacelike axis in $E_{1}^{4}$ can be parametrized with respect to the mean curvature by

$\Gamma(x, y, z)=\left(x \cosh y \cosh z, x \sinh y, x \cosh y \sinh z, \pm \int \frac{3 \int_{1}^{x} H(t) t^{2} d t+c_{2}}{\sqrt{x^{4}+\left(3 \int_{1}^{x} H(t) t^{2} d t+c_{2}\right)^{2}}} d x\right)$,

where $c_{2} \in \mathbb{R}$.

Example 2.8. If we take $H(x)=\frac{-2}{3 x}$ and $c_{2}=-1$ in $(2.24)$, then the rotational hypersurface is

$$
\Gamma(x, y, z)=\left(x \cosh y \cosh z, x \sinh y, x \cosh y \sinh z, \frac{-x}{\sqrt{2}}\right) .
$$

In Figure 2, one can see the projections of the rotational hypersurface (2.25) for $z=2$ into $x_{2} x_{3} x_{4}, x_{1} x_{3} x_{4}, x_{1} x_{2} x_{4}$ and $x_{1} x_{2} x_{3}$-spaces in (a), (b), (c) and (d), respectively.

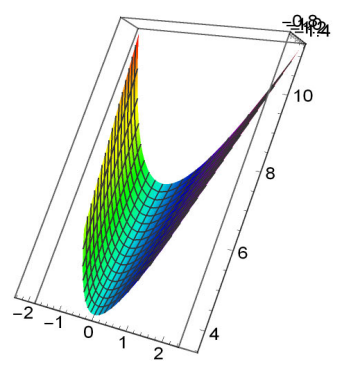

(a)

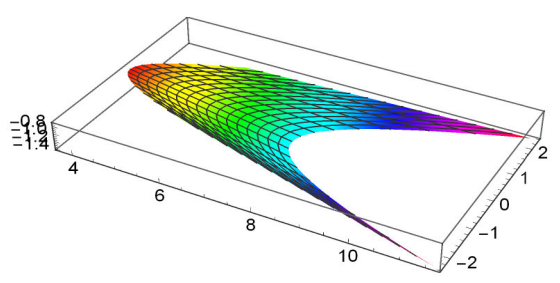

(c)

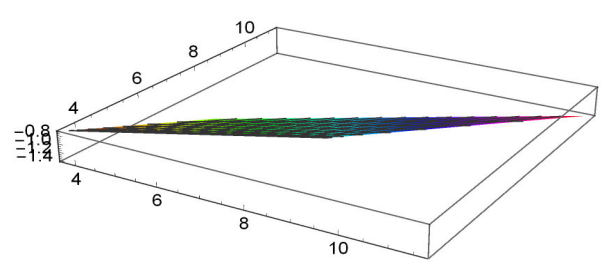

(b)

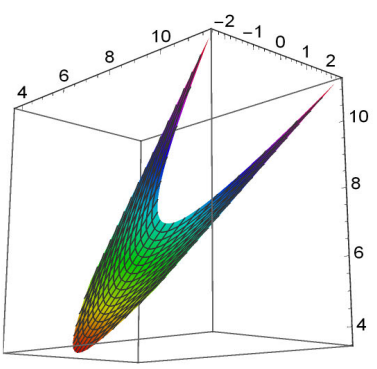

(d)

Figure 2 


\subsection{Gauss map of the rotational hypersurface about spacelike axis in $E_{1}^{4}$}

From (2.2), let us parametrize the Gauss map of the rotational hypersurface (2.1) about spacelike axis in $E_{1}^{4}$ as

$$
\Gamma_{G}(x, y, z)=-\frac{1}{\sqrt{1-f^{\prime 2}}}\left(f^{\prime} \cosh y \cosh z, f^{\prime} \sinh y, f^{\prime} \cosh y \sinh z, 1\right) .
$$

Then, from (1.4) the normal of (2.26) is

$$
N_{G}=\frac{1}{\sqrt{1-f^{\prime 2}}}\left(f^{\prime} \cosh y \cosh z, f^{\prime} \sinh y, f^{\prime} \cosh y \sinh z, 1\right)
$$

and from (2.27), we have

$$
\left\langle N_{G}, N_{G}\right\rangle=1
$$

From (1.5), we obtain the matrix of the first fundamental form, its inverse and determinant as

$$
\begin{gathered}
{\left[g_{i j}\right]_{G}=\frac{1}{1-f^{\prime 2}}\left[\begin{array}{ccc}
\frac{-f^{\prime 2}}{\left(1-f^{\prime 2}\right)} & 0 & 0 \\
0 & f^{\prime 2} & 0 \\
0 & 0 & f^{\prime 2} \cosh ^{2} y
\end{array}\right],} \\
{\left[g^{i j}\right]_{G}=\left(-1+f^{\prime 2}\right)\left[\begin{array}{ccc}
-\frac{\left(-1+f^{\prime 2}\right)}{f^{\prime 2}} & 0 & 0 \\
0 & -\frac{1}{f^{\prime 2}} & 0 \\
0 & 0 & -\frac{1}{f^{\prime 2} \cosh ^{2} y}
\end{array}\right]}
\end{gathered}
$$

and

$$
\operatorname{det}\left(\left[g_{i j}\right]_{G}\right)=-\frac{f^{\prime 4} f^{\prime \prime 2} \cosh ^{2} y}{\left(f^{\prime 2}-1\right)^{4}},
$$

respectively. From (1.6), the matrix form of the second fundamental form of (2.26), its inverse and determinant as

$$
\begin{gathered}
{\left[h_{i j}\right]_{G}=-\frac{1}{f^{\prime 2}-1}\left[\begin{array}{ccc}
\frac{f^{\prime \prime 2}}{\left(f^{\prime 2}-1\right)} & 0 & 0 \\
0 & f^{\prime 2} & 0 \\
0 & 0 & f^{\prime 2} \cosh ^{2} y
\end{array}\right],} \\
{\left[h^{i j}\right]_{G}=\left(f^{\prime 2}-1\right)\left[\begin{array}{ccc}
-\frac{\left(f^{\prime 2}-1\right)}{f^{\prime \prime 2}} & 0 & 0 \\
0 & -\frac{1}{f^{\prime 2}} & 0 \\
0 & 0 & -\frac{1}{f^{\prime 2} \cosh ^{2} y}
\end{array}\right]}
\end{gathered}
$$

and

$$
\operatorname{det}\left(\left[h_{i j}\right]_{G}\right)=-\frac{f^{\prime 4} f^{\prime \prime 2} \cosh ^{2} y}{\left(f^{\prime 2}-1\right)^{4}},
$$

respectively. Hence, using (2.28), (2.31) and (2.34) in (1.8), we have

Theorem 2.9. The Gaussian curvature of (2.26) is

$$
K_{G}=1 .
$$

Also, using (2.30) and (2.32) in (1.7), the shape operator of (2.26) is obtained by

$$
S_{G}=\left[\begin{array}{lll}
1 & 0 & 0 \\
0 & 1 & 0 \\
0 & 0 & 1
\end{array}\right] .
$$

So, from (1.9), (2.28) and (2.36), we get

Theorem 2.10. The mean curvature of (2.26) is

$$
H_{G}=1 \text {. }
$$




\subsection{The second Laplace-Beltrami operator on rotational hypersurface about spacelike axis in $E_{1}^{4}$}

The second Laplace-Beltrami $\left(\mathrm{LB}^{\mathrm{II}}\right)$ operator of a smooth function $\varphi=\left.\varphi\left(x^{1}, x^{2}, x^{3}\right)\right|_{D}$, $\left(D \subset \mathbb{R}^{3}\right.$ ) of class $C^{3}$ with respect to the nondegenerate second fundamental form of hypersurface $\Gamma$ is the operator which is defined as follows:

$$
\Delta^{I I} \varphi=-\frac{1}{\sqrt{\left|\operatorname{det}\left[h_{i j}\right]\right|}} \sum_{i, j=1}^{3} \frac{\partial}{\partial x^{i}}\left(\sqrt{\left|\operatorname{det}\left[h_{i j}\right]\right|} h^{i j} \frac{\partial \varphi}{\partial x^{j}}\right),
$$

where $h^{i j}$ are the components of the matrix $\left[h_{i j}\right]^{-1}$. So, using (1.11), (1.12) and (2.38), the $\mathrm{LB}^{\mathrm{II}}$ operator of a smooth function $\varphi=\varphi(x, y, z)$ can be written as

$$
\Delta^{I I} \varphi=-\frac{1}{\sqrt{\left|\operatorname{det}\left[h_{i j}\right]\right|}}\left\{\begin{array}{c}
\frac{\partial}{\partial x}\left(\frac{\left(h_{22} h_{33}-h_{23} h_{32}\right) \varphi_{x}+\left(h_{13} h_{32}-h_{12} h_{33}\right) \varphi_{y}+\left(h_{12} h_{23}-h_{13} h_{22}\right) \varphi_{z}}{\sqrt{\left|\operatorname{det}\left[h_{i j}\right]\right|}}\right) \\
+\frac{\partial}{\partial y}\left(\frac{\left(h_{23} h_{31}-h_{21} h_{33}\right) \varphi_{x}+\left(h_{11} h_{33}-h_{13} h_{31}\right) \varphi_{y}+\left(h_{13} h_{21}-h_{11} h_{23}\right) \varphi_{z}}{\sqrt{\left|\operatorname{det}\left[h_{i j}\right]\right|}}\right) \\
+\frac{\partial}{\partial z}\left(\frac{\left(h_{21} h_{32}-h_{22} h_{31}\right) \varphi_{x}+\left(h_{12} h_{31}-h_{11} h_{32}\right) \varphi_{y}+\left(h_{11} h_{22}-h_{12} h_{21}\right) \varphi_{z}}{\sqrt{\left|\operatorname{det}\left[h_{i j}\right]\right|}}\right)
\end{array}\right\},
$$

where

$$
\operatorname{det}\left[h_{i j}\right]=-h_{13} h_{22} h_{31}+h_{12} h_{23} h_{31}+h_{13} h_{21} h_{32}-h_{11} h_{23} h_{32}-h_{12} h_{21} h_{33}+h_{11} h_{22} h_{33} \text {. }
$$

Now, if we denote the $\mathrm{LB}^{\mathrm{II}}$ operator of the rotational hypersurface (2.1) in $E_{1}^{4}$ as $\Delta^{I I} \Gamma$, then from (2.1) and (2.39), we get

$$
\begin{aligned}
\Delta^{I I} \Gamma & =\left(\left(\Delta^{I I} \Gamma\right)_{1},\left(\Delta^{I I} \Gamma\right)_{2},\left(\Delta^{I I} \Gamma\right)_{3},\left(\Delta^{I I} \Gamma\right)_{4}\right) \\
& =-\frac{1}{\sqrt{\left|\operatorname{det}\left[h_{i j}\right]\right|}}\left(\begin{array}{c}
\left(U_{1}\right)_{x}+\left(V_{1}\right)_{y}+\left(W_{1}\right)_{z},\left(U_{2}\right)_{x}+\left(V_{2}\right)_{y}+\left(W_{2}\right)_{z}, \\
\left(U_{3}\right)_{x}+\left(V_{3}\right)_{y}+\left(W_{3}\right)_{z},\left(U_{4}\right)_{x}+\left(V_{4}\right)_{y}+\left(W_{4}\right)_{z}
\end{array}\right),
\end{aligned}
$$

where

$$
\left.\begin{array}{rl}
U_{i} & =\frac{1}{\sqrt{\left|\operatorname{det}\left[h_{i j}\right]\right|}}\left(\left(h_{22} h_{33}-h_{23} h_{32}\right)\left(\Gamma_{i}\right)_{x}+\left(h_{13} h_{32}-h_{12} h_{33}\right)\left(\Gamma_{i}\right)_{y}+\left(h_{12} h_{23}-h_{13} h_{22}\right)\left(\Gamma_{i}\right)_{z}\right), \\
V_{i} & =\frac{1}{\sqrt{\left|\operatorname{det}\left[h_{i j}\right]\right|}}\left(\left(h_{23} h_{31}-h_{21} h_{33}\right)\left(\Gamma_{i}\right)_{x}+\left(h_{11} h_{33}-h_{13} h_{31}\right)\left(\Gamma_{i}\right)_{y}+\left(h_{13} h_{21}-h_{11} h_{23}\right)\left(\Gamma_{i}\right)_{z}\right), \\
W_{i} & =\frac{1}{\sqrt{\mid \operatorname{det}\left[h_{i j}||\right.}}\left(\left(h_{21} h_{32}-h_{22} h_{31}\right)\left(\Gamma_{i}\right)_{x}+\left(h_{12} h_{31}-h_{11} h_{32}\right)\left(\Gamma_{i}\right)_{y}+\left(h_{11} h_{22}-h_{12} h_{21}\right)\left(\Gamma_{i}\right)_{z}\right) .
\end{array}\right\}
$$

Here, taking $i=1,2,3,4$ and using (2.1), (2.7)-(2.9), we have

$$
\left.\begin{array}{c}
U_{1}=\frac{x f^{\prime} \cosh ^{2} y \cosh z}{\sqrt{-f^{\prime \prime} \sqrt{1-f^{\prime 2}}}}, U_{2}=\frac{x f^{\prime} \sinh y \cosh y}{\sqrt{-f^{\prime \prime} \sqrt{1-f^{\prime 2}}}}, \\
U_{3}=\frac{x f^{\prime} \cosh ^{2} y \sinh z}{\sqrt{-f^{\prime \prime} \sqrt{1-f^{\prime 2}}}}, U_{4}=\frac{x f^{\prime 2} \cosh y}{\sqrt{-f^{\prime \prime} \sqrt{1-f^{\prime 2}}}} ;
\end{array}\right\}
$$

and

$$
\left.\begin{array}{l}
W_{1}=-\frac{x f^{\prime \prime} \sinh z}{\sqrt{-f^{\prime \prime} \sqrt{1-f^{\prime 2}}}}, W_{2}=0 \\
W_{3}=-\frac{x f^{\prime \prime} \cosh z}{\sqrt{-f^{\prime \prime} \sqrt{1-f^{\prime 2}}}}, W_{4}=0
\end{array}\right\}
$$


Thus, using (2.43)-(2.45) in (2.41), we obtain the components of the $\mathrm{LB}^{\mathrm{II}}$ operator of the rotational hypersurface $(2.1)$ as

$$
\left.\begin{array}{l}
\left(\Delta^{I I} \Gamma\right)_{1}=-\frac{\left(x f^{\prime \prime 2}\left(2-3 f^{\prime 2}\right)-f^{\prime}\left(2 f^{\prime \prime}-x f^{\prime \prime \prime}\right)\left(1-f^{\prime 2}\right)\right) \cosh y \cosh z}{2 x f^{\prime} f^{\prime \prime 2} \sqrt{1-f^{\prime 2}}}, \\
\left(\Delta^{I I} \Gamma\right)_{2}=-\frac{\left(x f^{\prime \prime 2}\left(2-3 f^{\prime 2}\right)-f^{\prime}\left(2 f^{\prime \prime}-x f^{\prime \prime \prime}\right)\left(1-f^{\prime 2}\right)\right) \sinh y}{2 x f^{\prime} f^{\prime \prime 2} \sqrt{1-f^{\prime 2}}}, \\
\left(\Delta^{I I} \Gamma\right)_{3}=-\frac{\left(x f^{\prime \prime 2}\left(2-3 f^{\prime 2}\right)-f^{\prime}\left(2 f^{\prime \prime}-x f^{\prime \prime \prime}\right)\left(1-f^{\prime 2}\right)\right) \cosh y \sinh z}{2 x f^{\prime} f^{\prime \prime 2} \sqrt{1-f^{\prime 2}}}, \\
\left(\Delta^{I I} \Gamma\right)_{4}=\frac{x f^{\prime \prime 2}\left(4-3 f^{\prime 2}\right)+f^{\prime}\left(2 f^{\prime \prime}-x f^{\prime \prime \prime}\right)\left(1-f^{\prime 2}\right)}{2 x f^{\prime \prime 2} \sqrt{1-f^{\prime 2}}},
\end{array}\right\}
$$

where $f(x) \neq a x+b, a, b \in \mathbb{R}$ and $f^{\prime \prime \prime}=\frac{d^{3} f(x)}{d x^{3}}$. So, we can give the following theorem:

Theorem 2.11. The rotational hypersurface (2.1) about spacelike axis in $E_{1}^{4}$ is not $L B^{I I}{ }_{-}$ minimal.

Proof. We know that, a hypersurface $\Gamma$ is $\mathrm{LB}^{\mathrm{II}}$-minimal if it satisfies $\Delta^{I I} \Gamma=0$. So, the rotational hypersurface $(2.1)$ in $E_{1}^{4}$ is $\mathrm{LB}^{\mathrm{II}}$-minimal, if all components of the $\mathrm{LB}^{\mathrm{II}}$ operator $\Delta^{I I} \Gamma$ vanishes, i.e. $\left(\Delta^{I I} \Gamma\right)_{i}, i=1,2,3,4$, which have been obtained in $(2.46)$ vanish identically. Hence, the solution of $\left(\Delta^{I I} \Gamma\right)_{i}=0, i=1,2,3$, in $(2.46)$ is obtained with the Mathematica as

$f(x)=\int_{1}^{x}$ InverseFunct $\left[\frac{1}{8}\left(\# 1\left(2 \# 1^{2}-1\right) \sqrt{1-\# 1^{2}}+\sin ^{-1}(\# 1)\right) \&\right]\left[c_{2}-\frac{1}{3} c_{1} t^{3}\right] d t+c_{3}$

and since this solution doesn't satisfy $\left(\Delta^{I I} \Gamma\right)_{4}=0$ in $(2.46)$, this hypersurface cannot be $\mathrm{LB}^{\mathrm{II}}$-minimal.

\subsection{The third Laplace-Beltrami operator on rotational hypersurface about spacelike axis in $E_{1}^{4}$}

The third Laplace-Beltrami $\left(\mathrm{LB}^{\mathrm{III}}\right)$ operator of a smooth function $\varphi=\left.\varphi\left(x^{1}, x^{2}, x^{3}\right)\right|_{D}$, $\left(D \subset R^{3}\right)$ of class $C^{3}$ with respect to the nondegenerate third fundamental form of hypersurface $\Gamma$ is the operator which is defined as follows:

$$
\Delta^{I I I} \varphi=\frac{1}{\sqrt{\left|\operatorname{det}\left[m_{i j}\right]\right|}} \sum_{i, j=1}^{3} \frac{\partial}{\partial x^{i}}\left(\sqrt{\left|\operatorname{det}\left[m_{i j}\right]\right|} m^{i j} \frac{\partial \varphi}{\partial x^{j}}\right),
$$

where $m^{i j}$ are the components of the matrix $\left(m_{i j}\right)^{-1}$. Here, the matrix of third fundamental form, its inverse and the determinant are obtained by

$$
\begin{gathered}
{\left[m_{i j}\right]=\left[\begin{array}{ccc}
m_{11} & m_{12} & m_{13} \\
m_{21} & m_{22} & m_{23} \\
m_{31} & m_{32} & m_{33}
\end{array}\right]=-\frac{1}{-1+f^{\prime 2}}\left[\begin{array}{ccc}
\frac{f^{\prime 2}}{-1+f^{\prime 2}} & 0 & 0 \\
0 & f^{\prime 2} & 0 \\
0 & 0 & f^{\prime 2} \cosh ^{2} y
\end{array}\right],} \\
{\left[m^{i j}\right]=\left(-1+f^{\prime 2}\right)\left[\begin{array}{ccc}
-\frac{\left(-1+f^{\prime 2}\right)}{f^{\prime \prime 2}} & 0 & 0 \\
0 & -\frac{1}{f^{\prime 2}} & 0 \\
0 & 0 & -\frac{1}{f^{\prime 2} \cosh ^{2} y}
\end{array}\right]}
\end{gathered}
$$

and

$$
\operatorname{det}\left[m_{i j}\right]=-\frac{f^{\prime 4} f^{\prime \prime 2} \cosh ^{2} y}{\left(-1+f^{\prime 2}\right)^{4}}
$$


respectively. Here, $m_{i j}=\left\langle\left(N_{\Gamma}\right)_{u_{i}},\left(N_{\Gamma}\right)_{u_{j}}\right\rangle$. So, using (1.11), (1.12) and (2.47) the LB $\mathrm{LB}^{\mathrm{III}}$ operator of a smooth function $\varphi=\varphi(x, y, z)$ can be written as

$\Delta^{I I I} \varphi=-\frac{1}{\sqrt{\left|\operatorname{det}\left[m_{i j}\right]\right|}}\left\{\begin{array}{c}\frac{\partial}{\partial x}\left(\frac{\left(m_{22} m_{33}-m_{23} m_{32}\right) \varphi_{x}+\left(m_{13} m_{32}-m_{12} m_{33}\right) \varphi_{y}+\left(m_{12} m_{23}-m_{13} m_{22}\right) \varphi_{z}}{\sqrt{\left|\operatorname{det}\left[m_{i j}\right]\right|}}\right) \\ +\frac{\partial}{\partial y}\left(\frac{\left(m_{23} m_{31}-m_{21} m_{33}\right) \varphi_{x}+\left(m_{11} m_{33}-m_{13} m_{31}\right) \varphi_{y}+\left(m_{13} m_{21}-m_{11} m_{23}\right) \varphi_{z}}{\sqrt{\left|\operatorname{det}\left[m_{i j}\right]\right|}}\right) \\ +\frac{\partial}{\partial z}\left(\frac{\left(m_{21} m_{32}-m_{22} m_{31}\right) \varphi_{x}+\left(m_{12} m_{31}-m_{11} m_{32}\right) \varphi_{y}+\left(m_{11} m_{22}-m_{12} m_{21}\right) \varphi_{z}}{\sqrt{\left|\operatorname{det}\left[m_{i j}\right]\right|}}\right)\end{array}\right\}$,

where

$\operatorname{det}\left[m_{i j}\right]=-m_{13} m_{22} m_{31}+m_{12} m_{23} m_{31}+m_{13} m_{21} m_{32}-m_{11} m_{23} m_{32}-m_{12} m_{21} m_{33}+m_{11} m_{22} m_{33}$.

Now, if we denote the $\mathrm{LB}^{\mathrm{III}}$ operator of the rotational hypersurface (2.1) in $E_{1}^{4}$ as $\Delta^{I I I} \Gamma$, then from (2.51), we get

$$
\begin{aligned}
\Delta^{I I I} \Gamma & =\left(\left(\Delta^{I I I} \Gamma\right)_{1},\left(\Delta^{I I I} \Gamma\right)_{2},\left(\Delta^{I I I} \Gamma\right)_{3},\left(\Delta^{I I I} \Gamma\right)_{4}\right) \\
& =-\frac{1}{\sqrt{\left|\operatorname{det}\left[m_{i j}\right]\right|}}\left(\begin{array}{c}
\left(\mathfrak{U}_{1}\right)_{x}+\left(\mathfrak{V}_{1}\right)_{y}+\left(\mathfrak{W}_{1}\right)_{z},\left(\mathfrak{U}_{2}\right)_{x}+\left(\mathfrak{V}_{2}\right)_{y}+\left(\mathfrak{W}_{2}\right)_{z}, \\
\left(\mathfrak{U}_{3}\right)_{x}+\left(\mathfrak{V}_{3}\right)_{y}+\left(\mathfrak{W}_{3}\right)_{z},\left(\mathfrak{U}_{4}\right)_{x}+\left(\mathfrak{V}_{4}\right)_{y}+\left(\mathfrak{W}_{4}\right)_{z}
\end{array}\right),
\end{aligned}
$$

where

$$
\left.\begin{array}{rl}
\mathfrak{U}_{i} & =\frac{\left(\left(m_{22} m_{33}-m_{23} m_{32}\right)\left(\Gamma_{i}\right)_{x}+\left(m_{13} m_{32}-m_{12} m_{33}\right)\left(\Gamma_{i}\right)_{y}+\left(m_{12} m_{23}-m_{13} m_{22}\right)\left(\Gamma_{i}\right)_{z}\right)}{\sqrt{\left|\operatorname{det}\left[m_{i j}\right]\right|}} \\
\mathfrak{V}_{i} & =\frac{\left(\left(m_{23} m_{31}-m_{21} m_{33}\right)\left(\Gamma_{i}\right)_{x}+\left(m_{11} m_{33}-m_{13} m_{31}\right)\left(\Gamma_{i}\right)_{y}+\left(m_{13} m_{21}-m_{11} m_{23}\right)\left(\Gamma_{i}\right)_{z}\right)}{\sqrt{\left|\operatorname{det}\left[m_{i j}\right]\right|}} \\
\mathfrak{W}_{i} & =\frac{\left(\left(m_{21} m_{32}-m_{22} m_{31}\right)\left(\Gamma_{i}\right)_{x}+\left(m_{12} m_{31}-m_{11} m_{32}\right)\left(\Gamma_{i}\right)_{y}+\left(m_{11} m_{22}-m_{12} m_{21}\right)\left(\Gamma_{i}\right)_{z}\right)}{\sqrt{\left|\operatorname{det}\left[m_{i j}\right]\right|}}
\end{array}\right\}
$$

Here, taking $i=1,2,3,4$ and using (2.1), (2.48)-(2.50), we have

$$
\left.\begin{array}{rl}
\mathfrak{U}_{1} & =\frac{f^{\prime 2} \cosh ^{2} y \cosh z}{f^{\prime \prime}}, \mathfrak{U}_{2}=\frac{f^{\prime 2} \sinh y \cosh y}{f^{\prime \prime}}, \\
\mathfrak{U}_{3} & =\frac{f^{\prime 2} \cosh ^{2} y \sinh z}{f^{\prime \prime}}, \mathfrak{U}_{4}=\frac{f^{\prime 3} \cosh y}{f^{\prime \prime}} ;
\end{array}\right\}
$$

and

$$
\left.\begin{array}{l}
\mathfrak{W}_{1}=-\frac{x f^{\prime \prime} \sinh z}{1-f^{\prime 2}}, \mathfrak{W}_{2}=0, \\
\mathfrak{W}_{3}=-\frac{x f^{\prime \prime} \cosh z}{1-f^{\prime 2}}, \mathfrak{W}_{4}=0 .
\end{array}\right\}
$$

Thus, using (2.55)-(2.57) in (2.53), we obtain the components of the LB ${ }^{\mathrm{III}}$ operator of the rotational hypersurface $(2.1)$ as

$$
\left.\begin{array}{l}
\left(\Delta^{I I I} \Gamma\right)_{1}=-\frac{\left(1-f^{\prime 2}\right)\left(\left(1-f^{\prime 2}\right) f^{\prime}\left(2 f^{\prime \prime 2}-f^{\prime} f^{\prime \prime \prime}\right)-2 x f^{\prime \prime 3}\right) \cosh y \cosh z}{f^{\prime 2} f^{\prime \prime 3}}, \\
\left(\Delta^{I I I} \Gamma\right)_{2}=-\frac{\left(1-f^{\prime 2}\right)\left(\left(1-f^{\prime 2}\right) f^{\prime}\left(2 f^{\prime \prime 2}-f^{\prime 2} f^{\prime \prime \prime}\right)-2 x f^{\prime \prime 3}\right) \sinh y}{f^{\prime 2} f^{\prime \prime 3}}, \\
\left(\Delta^{I I I} \Gamma\right)_{3}=-\frac{\left(1-f^{\prime 2}\right)\left(\left(1-f^{\prime 2}\right) f^{\prime}\left(f^{\prime \prime 2}-f^{\prime} f^{\prime \prime \prime}\right)-2 x f^{\prime \prime 3}\right) \cosh y \sinh z}{f^{\prime 2}(x) f^{\prime \prime 3}(x)} \\
\left(\Delta^{I I I} \Gamma\right)_{4}=-\frac{\left(-1+f^{\prime 2}\right)^{2}\left(3 f^{\prime \prime 2}-f^{\prime} f^{\prime \prime \prime}\right)}{f^{\prime \prime 3}},
\end{array}\right\}
$$

where $f(x) \neq a x+b, a, b \in \mathbb{R}$. So,

Theorem 2.12. The rotational hypersurface

$$
\Gamma(x, y, z)=\left(x \cosh y \cosh z, x \sinh y, x \cosh y \sinh z, d_{2} \sqrt{2 x+\left(d_{2}\right)^{2}}+d_{3}\right), d_{2}, d_{3} \in \mathbb{R}
$$

about spacelike axis in $E_{1}^{4}$ is $L B^{I I I}$-minimal. 
Proof. We know that, a hypersurface $\Gamma$ is $\mathrm{LB}^{\mathrm{III}}$-minimal if it satisfies $\Delta^{I I I} \Gamma=0$. So, the rotational hypersurface $(2.1)$ in $E_{1}^{4}$ is $\mathrm{LB}^{\mathrm{III}}$-minimal, if all components of the LB ${ }^{\mathrm{III}}$ operator $\Delta^{I I I} \Gamma$ vanishes, i.e. $\left(\Delta^{I I I} \Gamma\right)_{i}, i=1,2,3,4$, which have been obtained in (2.58) vanish identically. Hence, the solution of the differential equation

$$
3 f^{\prime \prime}(x)^{2}-f^{\prime}(x) f^{\prime \prime \prime}(x)=0
$$

is obtained as

$$
f(x)=\sqrt{2 x+d_{1}} d_{2}+d_{3},
$$

$d_{1}, d_{2}, d_{3} \in \mathbb{R}$. If we use this function in the first three components of $\mathrm{LB}^{\mathrm{III}}$ operator $\Delta^{I I I} \Gamma$, then for these components to be zero, it must be $d_{1}=\left(d_{2}\right)^{2}$ and this completes the proof.

\section{Rotational hypersurfaces about timelike axis in $E_{1}^{4}$}

In this section, we find the rotational hypersurface about timelike axis according to the Gaussian and mean curvatures in $E_{1}^{4}$ and give some results for the Gauss map of this hypersurface. Also, we study the $\mathrm{LB}^{\mathrm{II}}$ and $\mathrm{LB}^{\mathrm{III}}$ operators on the rotational hypersurface with timelike axis in $E_{1}^{4}$ and give some characterizations for $\mathrm{LB}^{\mathrm{II}}$-minimality and $\mathrm{LB}^{\mathrm{III}}$ minimality of this hypersurface.

\subsection{Curvatures of rotational hypersurfaces about timelike axis in $E_{1}^{4}$}

For a differentiable function $g(x): I \subset \mathbb{R} \longrightarrow \mathbb{R}$, the rotational hypersurface which is obtained by rotating the profile curve $\beta(x)=(g(x), 0,0, x)$ about timelike axis $(1,0,0,0)$ is given by

$$
\begin{aligned}
\Gamma(x, y, z) & =\left[\begin{array}{cccc}
1 & 0 & 0 & 0 \\
0 & \cos z & -\sin y \sin z & -\cos y \sin z \\
0 & 0 & \cos y & -\sin y \\
0 & \sin z & \sin y \cos z & \cos y \cos z
\end{array}\right] \cdot\left[\begin{array}{c}
g(x) \\
0 \\
0 \\
x
\end{array}\right] \\
& =(g(x),-x \cos y \sin z,-x \sin y, x \cos y \cos z),
\end{aligned}
$$

where $x \in \mathbb{R}-\{0\}$ and $0 \leq y, z \leq 2 \pi$.

With the aid of the first differentials of (3.1) with respect to $x, y$ and $z$, the Gauss map of the rotational hypersurface (3.1) is obtained from (1.4) by

$$
N_{\Gamma}=\frac{1}{\sqrt{g^{\prime 2}-1}}\left(1,-g^{\prime} \cos y \sin z,-g^{\prime} \sin y, g^{\prime} \cos y \cos z\right)
$$

and from (3.2), we get

$$
\left\langle N_{\Gamma}, N_{\Gamma}\right\rangle=1 .
$$

Here, we state $g=g(x)$ and $g^{\prime}=\frac{d g(x)}{d x}$.

Also, from (1.5), we obtain the matrix of the first fundamental form, its inverse and determinant as

and

$$
\begin{gathered}
{\left[g_{i j}\right]=\left[\begin{array}{ccc}
1-g^{\prime 2} & 0 & 0 \\
0 & x^{2} & 0 \\
0 & 0 & x^{2} \cos ^{2} y
\end{array}\right],} \\
{\left[g^{i j}\right]=\left[\begin{array}{ccc}
\frac{1}{1-g^{\prime 2}} & 0 & 0 \\
0 & \frac{1}{x^{2}} & 0 \\
0 & 0 & \frac{1}{x^{2} \cos ^{2} y}
\end{array}\right]}
\end{gathered}
$$

$$
\operatorname{det}\left[g_{i j}\right]=-x^{4}\left(g^{\prime 2}-1\right) \cos ^{2} y,
$$


respectively. From (1.6), the matrix form of the second fundamental form of the hypersurface (3.1), its inverse and determinant as

$$
\begin{gathered}
{\left[h_{i j}\right]=-\frac{1}{\sqrt{g^{\prime 2}-1}}\left[\begin{array}{ccc}
g^{\prime \prime} & 0 & 0 \\
0 & x g^{\prime} & 0 \\
0 & 0 & x g^{\prime} \cos ^{2} y
\end{array}\right],} \\
{\left[h^{i j}\right]=\sqrt{g^{\prime 2}-1}\left[\begin{array}{ccc}
-\frac{1}{g^{\prime \prime}} & 0 & 0 \\
0 & -\frac{1}{x g^{\prime}} & 0 \\
0 & 0 & -\frac{1}{x g^{\prime} \cos ^{2} y}
\end{array}\right]}
\end{gathered}
$$

and

$$
\operatorname{det}\left[h_{i j}\right]=-\frac{x^{2} g^{\prime 2} g^{\prime \prime} \cos ^{2} y}{\left(g^{\prime 2}-1\right)^{3 / 2}},
$$

respectively and here, we state $g^{\prime \prime}=\frac{d^{2} g(x)}{d x^{2}}$. Hence, using (3.3), (3.6) and (3.9) in (1.8), we can give the following theorem:

Theorem 3.1. The Gaussian curvature of the rotational hypersurface (3.1) is

$$
K=\frac{g^{\prime 2} g^{\prime \prime}}{x^{2}\left(g^{\prime 2}-1\right)^{\frac{5}{2}}} .
$$

Here, we want to find the function $g$ according to the Gaussian curvature $K$ by solving the equation (3.10). For solving the differential equation (3.10), let us put

$$
C=\frac{g^{\prime 3}}{x^{6}\left(g^{\prime 2}-1\right)^{3 / 2}}
$$

By differentiating (3.11) and using (3.10), we have

$$
C^{\prime}=-\frac{3 K(x)+6 x^{3} C}{x^{4}} .
$$

The solution of (3.12) which is a first order differential equation with respect to $C$ is obtained by

$$
C=\frac{c_{3}-3 \int_{1}^{x} K(t) t^{2} d t}{x^{6}}
$$

$c_{3} \in \mathbb{R}$. From (3.11) and (3.13), we obtain that

$$
g(x)= \pm \int \frac{\left(c_{3}-3 \int_{1}^{x} K(t) t^{2} d t\right)^{\frac{1}{3}}}{\sqrt{\left(c_{3}-3 \int_{1}^{x} K(t) t^{2} d t\right)^{\frac{2}{3}}-1}} d x .
$$

Hence, we can state the following theorem:

Theorem 3.2. The rotational hypersurface (3.1) about timelike axis in $E_{1}^{4}$ can be parametrized according to the Gaussian curvature by

$$
\Gamma(x, y, z)=\left( \pm \int \frac{\left(c_{3}-3 \int_{1}^{x} K(t) t^{2} d t\right)^{\frac{1}{3}}}{\sqrt{\left(c_{3}-3 \int_{1}^{x} K(t) t^{2} d t\right)^{\frac{2}{3}}-1}} d x,-x \cos y \sin z,-x \sin y, x \cos y \cos z\right)
$$

where $c_{3} \in \mathbb{R}$.

Example 3.3. If we take $K(x)=\frac{e^{3 x}}{x^{2}}$ and $c_{3}=-e^{3}$ in (3.15), then the rotational hypersurface is

$$
\Gamma(x, y, z)=\left(-\ln \left(e^{x}+\sqrt{e^{2 x}-1}\right),-x \cos y \sin z,-x \sin y, x \cos y \cos z\right) .
$$

In the following figures, one can see the projections of the rotational hypersurface (3.16) for $z=2$ into $x_{2} x_{3} x_{4}, x_{1} x_{3} x_{4}, x_{1} x_{2} x_{4}$ and $x_{1} x_{2} x_{3}$-spaces in (a), (b), (c) and (d), respectively. 


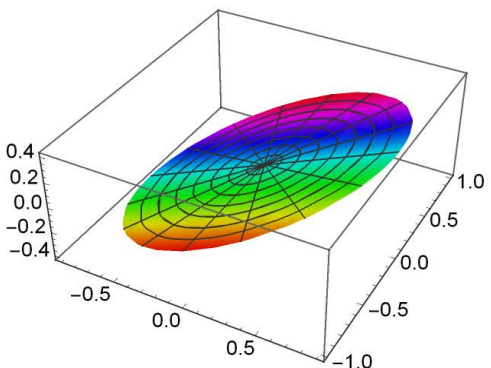

(a)

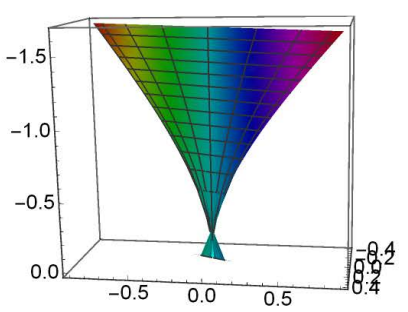

(c)

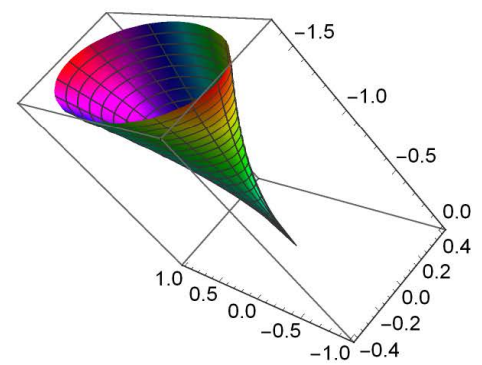

(b)

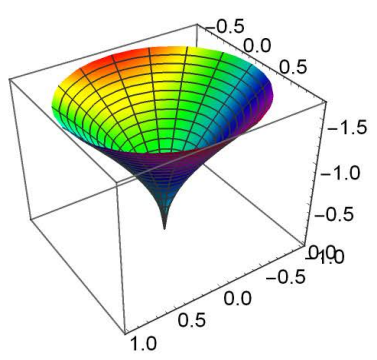

(d)

Figure 3

Also from Theorem 3.2, we can give the following results:

Corollary 3.4. The rotational hypersurface (3.1) about timelike axis in $E_{1}^{4}$ with constant Gaussian curvature $(K=k \in \mathbb{R})$ can be parametrized by

$$
\Gamma(x, y, z)=\left( \pm \int \frac{\left(c_{3}+k-k x^{3}\right)^{\frac{1}{3}}}{\sqrt{\left(c_{3}+k-k x^{3}\right)^{\frac{2}{3}}-1}} d x,-x \cos y \sin z,-x \sin y, x \cos y \cos z\right) .
$$

Corollary 3.5. The rotational hypersurface (3.1) about timelike axis in $E_{1}^{4}$ with zero Gaussian curvature can be parametrized by

$$
\Gamma(x, y, z)=\left( \pm \frac{x\left(c_{3}\right)^{\frac{1}{3}}}{\sqrt{\left(c_{3}\right)^{\frac{2}{3}}-1}},-x \cos y \sin z,-x \sin y, x \cos y \cos z\right) .
$$

Also, using (3.5) and (3.7) in (1.7), the shape operator of the rotational hypersurface (3.1) is obtained by

$$
S=\frac{1}{\sqrt{g^{\prime 2}-1}}\left[\begin{array}{ccc}
\frac{g^{\prime \prime}}{g^{\prime 2}-1} & 0 & 0 \\
0 & -\frac{g^{\prime}}{x} & 0 \\
0 & 0 & -\frac{g^{\prime}}{x}
\end{array}\right]
$$

So, from (1.9), (3.3) and (3.17), we get

Theorem 3.6. The mean curvature of the rotational hypersurface (3.1) is

$$
H=\frac{2 g^{\prime}\left(1-g^{\prime 2}\right)+x g^{\prime \prime}}{3 x\left(g^{\prime 2}-1\right)^{3 / 2}} .
$$

For solving the differential equation (3.18), let us take

$$
D=\frac{g^{\prime}(x)}{x \sqrt{g^{\prime 2}-1}} .
$$


By differentiating (3.19) and using (3.18), we have

$$
D^{\prime}=-\frac{3 D+3 H(x)}{x}
$$

The solution of (3.20) which is a first order differential equation with respect to $D$ is obtained by

$$
D=\frac{-3 \int_{1}^{x} H(t) t^{2} d t+c_{4}}{x^{3}}
$$

$c_{4} \in \mathbb{R}$. From (3.19) and (3.21), we obtain that

$$
g(x)= \pm \int \frac{-3 \int_{1}^{x} H(t) t^{2} d t+c_{4}}{\sqrt{\left(-3 \int_{1}^{x} H(t) t^{2} d t+c_{4}\right)^{2}-x^{4}}} d x .
$$

Thus, we can give the following theorem:

Theorem 3.7. The rotational hypersurface (3.1) about timelike axis in $E_{1}^{4}$ can be parametrized according to the mean curvature by

$\Gamma(x, y, z)=\left( \pm \int \frac{-3 \int_{1}^{x} H(t) t^{2} d t+c_{4}}{\sqrt{\left(-3 \int_{1}^{x} H(t) t^{2} d t+c_{4}\right)^{2}-x^{4}}} d x,-x \cos y \sin z,-x \sin y, x \cos y \cos z\right)$,

where $c_{4} \in \mathbb{R}$.

Example 3.8. If we take $H(x)=-\frac{2 \cot x-x \csc ^{2} x}{3 x}$ and $c_{4}=\cot (1)$ in (3.23), then the rotational hypersurface is

$$
\Gamma(x, y, z)=\left(\frac{\arcsin (\sqrt{2} \sin x)}{\sqrt{2}},-x \cos y \sin z,-x \sin y, x \cos y \cos z\right) .
$$

In the following figures, one can see the projections of the rotational hypersurface (3.24) for $z=2$ into $x_{2} x_{3} x_{4}, x_{1} x_{3} x_{4}, x_{1} x_{2} x_{4}$ and $x_{1} x_{2} x_{3}$-spaces in (a), (b), (c) and (d), respectively.

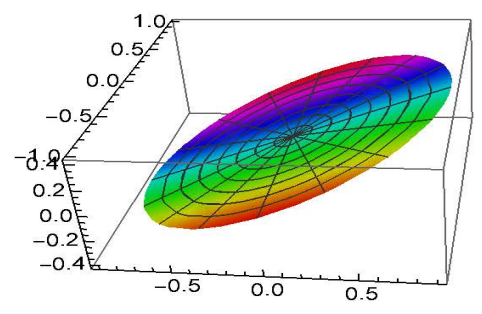

(a)

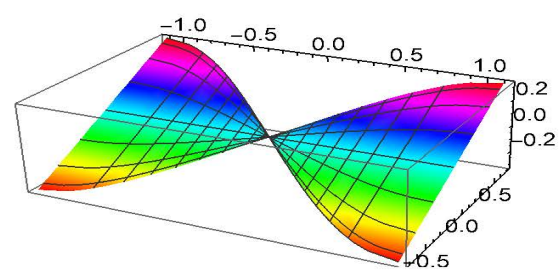

(c)

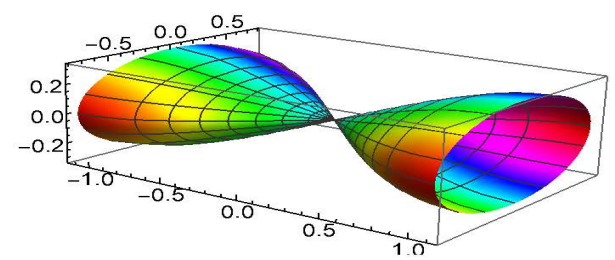

(b)

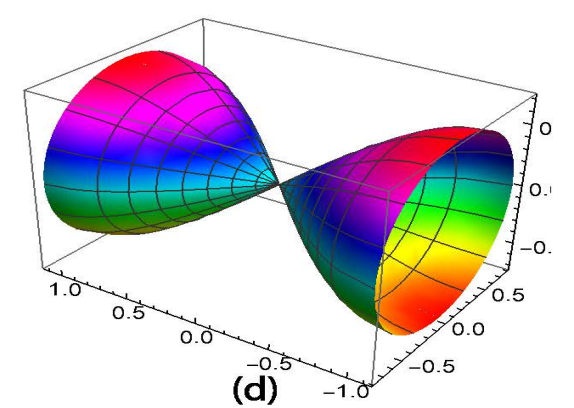

(d)

Figure 4 


\subsection{Gauss map of the rotational hypersurface about timelike axis in $E_{1}^{4}$}

From (3.2), let us parametrize the Gauss map of the rotational hypersurface (3.1) about timelike axis in $E_{1}^{4}$ as

$$
\Gamma_{G}(x, y, z)=\frac{1}{\sqrt{g^{\prime 2}-1}}\left(1,-g^{\prime} \cos y \sin z,-g^{\prime} \sin y, g^{\prime} \cos y \cos z\right) .
$$

Then, the normal of $(3.25)$ is

$$
N_{G}=-\frac{1}{\sqrt{g^{\prime 2}-1}}\left(1,-g^{\prime} \cos y \sin z,-g^{\prime} \sin y, g^{\prime} \cos y \cos z\right)
$$

and from (3.26), we have

$$
\left\langle N_{G}, N_{G}\right\rangle=1
$$

From (1.5), we obtain the matrix of the first fundamental form, its inverse and determinant as

$$
\begin{gathered}
{\left[g_{i j}\right]_{G}=\frac{1}{g^{\prime 2}-1}\left[\begin{array}{ccc}
\frac{-g^{\prime \prime 2}}{g^{\prime 2}-1} & 0 & 0 \\
0 & g^{\prime 2} & 0 \\
0 & 0 & g^{\prime 2} \cos ^{2} y
\end{array}\right],} \\
{\left[g^{i j}\right]_{G}=\left(g^{\prime 2}-1\right)\left[\begin{array}{ccc}
-\frac{g^{\prime 2}-1}{g^{\prime \prime 2}} & 0 & 0 \\
0 & \frac{1}{g^{\prime 2}} & 0 \\
0 & 0 & \frac{1}{g^{\prime 2} \cos ^{2} y}
\end{array}\right]}
\end{gathered}
$$

and

$$
\operatorname{det}\left(\left[g_{i j}\right]_{G}\right)=-\frac{g^{\prime 4} g^{\prime \prime 2} \cos ^{2} y}{\left(g^{\prime 2}-1\right)^{4}}
$$

respectively. From (1.6), the matrix form of the second fundamental form of (3.25), its inverse and determinant as

$$
\begin{gathered}
{\left[h_{i j}\right]_{G}=\frac{1}{g^{\prime 2}-1}\left[\begin{array}{ccc}
\frac{-g^{\prime 2}}{g^{\prime 2}-1} & 0 & 0 \\
0 & g^{\prime 2} & 0 \\
0 & 0 & g^{\prime 2} \cos ^{2} y
\end{array}\right],} \\
{\left[h^{i j}\right]_{G}=\left(g^{\prime 2}-1\right)\left[\begin{array}{ccc}
-\frac{g^{\prime 2}-1}{g^{\prime 2}} & 0 & 0 \\
0 & \frac{1}{g^{\prime 2}} & 0 \\
0 & 0 & \frac{1}{g^{\prime 2} \cos ^{2} y}
\end{array}\right]}
\end{gathered}
$$

and

$$
\operatorname{det}\left(\left[h_{i j}\right]_{G}\right)=-\frac{g^{\prime 4} g^{\prime \prime 2} \cos ^{2} y}{\left(g^{\prime 2}-1\right)^{4}}
$$

respectively. Hence, using (3.27), (3.30) and (3.33) in (1.8), we have

Theorem 3.9. The Gaussian curvature of (3.25) is

$$
K_{G}=1 .
$$

Also, using (3.29) and (3.31) in (1.7), the shape operator of (3.25) is obtained by

$$
S_{G}=\left[\begin{array}{lll}
1 & 0 & 0 \\
0 & 1 & 0 \\
0 & 0 & 1
\end{array}\right] .
$$

So, from (1.9), (3.27) and (3.35), we get

Theorem 3.10. The mean curvature of (3.25) is

$$
H_{G}=1 \text {. }
$$




\subsection{The second Laplace-Beltrami operator on rotational hypersurface about timelike axis in $E_{1}^{4}$}

Using the same procedure in subsection 2.3, we obtain the components $U_{i}, V_{i}$ and $W_{i}$ which are defined in (2.42) as

$$
\left.\begin{array}{c}
U_{1}=\frac{x g^{\prime 2} \cos y}{\sqrt{-g^{\prime \prime} \sqrt{g^{\prime 2}-1}}}, U_{2}=-\frac{x g^{\prime} \cos ^{2} y \sin z}{\sqrt{-g^{\prime \prime} \sqrt{g^{\prime 2}-1}}}, \\
U_{3}=-\frac{x g^{\prime} \sin y \cos y}{\sqrt{-g^{\prime \prime} \sqrt{g^{\prime 2}-1}}}, U_{4}=\frac{x g^{\prime} \cos ^{2} y \cos z}{\sqrt{-g^{\prime \prime} \sqrt{g^{\prime 2}-1}}} ;
\end{array}\right\}
$$

and

$$
\left.\begin{array}{l}
W_{1}=0, W_{2}=-\frac{x g^{\prime \prime} \cos z}{\sqrt{-g^{\prime \prime} \sqrt{g^{\prime 2}-1}}}, \\
W_{3}=0, W_{4}=-\frac{x g^{\prime \prime} \sin z}{\sqrt{-g^{\prime \prime} \sqrt{g^{\prime 2}-1}}} \cdot
\end{array}\right\}
$$

Thus, using (3.37)-(3.39) in (2.41), we obtain the components of the $\mathrm{LB}^{\mathrm{II}}$ operator of the rotational hypersurface $(3.1)$ as

$$
\left.\begin{array}{l}
\left(\Delta^{I I} \Gamma\right)_{1}=\frac{x g^{\prime \prime 2}\left(4-3 g^{\prime 2}\right)+g^{\prime}\left(2 g^{\prime \prime}-x g^{\prime \prime \prime}\right)\left(1-g^{\prime 2}\right)}{2 x g^{\prime \prime 2} \sqrt{g^{\prime 2}-1}}, \\
\left(\Delta^{I I} \Gamma\right)_{2}=\frac{\left(x g^{\prime \prime 2}\left(2-3 g^{\prime 2}\right)-g^{\prime}\left(2 g^{\prime \prime}-x g^{\prime \prime \prime}\right)\left(1-g^{\prime 2}\right)\right) \cos y \sin z}{2 x g^{\prime} g^{\prime \prime 2} \sqrt{g^{\prime 2}-1}}, \\
\left(\Delta^{I I} \Gamma\right)_{3}=\frac{\left(x g^{\prime \prime 2}\left(2-3 g^{\prime 2}\right)-g^{\prime}\left(2 g^{\prime \prime}-x g^{\prime \prime \prime}\right)\left(1-g^{\prime 2}\right)\right) \sin y}{2 x g^{\prime} g^{\prime \prime 2} \sqrt{g^{\prime 2}-1}}, \\
\left(\Delta^{I I} \Gamma\right)_{4}=-\frac{\left(x g^{\prime \prime 2}\left(2-3 g^{\prime 2}\right)-g^{\prime}\left(2 g^{\prime \prime}-x g^{\prime \prime \prime}\right)\left(1-g^{\prime 2}\right)\right) \cos y \cos z}{2 x g^{\prime} g^{\prime \prime 2} \sqrt{g^{\prime 2}-1}},
\end{array}\right\}
$$

where $g(x) \neq a x+b, a, b \in \mathbb{R}$ and $g^{\prime \prime \prime}=\frac{d^{3} g(x)}{d x^{3}}$. So, from the proof of the Theorem 2.11, we can state the following theorem:

Theorem 3.11. The rotational hypersurface (3.1) about timelike axis in $E_{1}^{4}$ is not $L B^{I I}$ minimal.

\subsection{The third Laplace-Beltrami operator on rotational hypersurface about timelike axis in $E_{1}^{4}$}

The matrix of third fundamental form, its inverse and the determinant are obtained by

$$
\begin{aligned}
& {\left[m_{i j}\right]=\frac{1}{g^{\prime 2}-1}\left[\begin{array}{ccc}
-\frac{g^{\prime \prime 2}}{g^{\prime 2}-1} & 0 & 0 \\
0 & g^{\prime 2} & 0 \\
0 & 0 & g^{\prime 2} \cos ^{2} y
\end{array}\right],} \\
& {\left[m^{i j}\right]=\left(g^{\prime 2}-1\right)\left[\begin{array}{ccc}
-\frac{g^{\prime 2}-1}{g^{\prime 2}} & 0 & 0 \\
0 & \frac{1}{g^{\prime 2}} & 0 \\
0 & 0 & \frac{1}{g^{\prime 2} \cos ^{2} y}
\end{array}\right]}
\end{aligned}
$$

and

$$
\operatorname{det}\left[m_{i j}\right]=-\frac{g^{\prime 4} g^{\prime \prime 2} \cos ^{2} y}{\left(g^{\prime 2}-1\right)^{4}}
$$


respectively. Using the same procedure in subsection 2.4, we obtain the components $\mathfrak{U}_{i}$, $\mathfrak{V}_{i}$ and $\mathfrak{W}_{i}$ which are defined in (2.54) as

$$
\left.\begin{array}{l}
\mathfrak{U}_{1}=\frac{g^{\prime 3} \cos y}{g^{\prime \prime}}, \mathfrak{U}_{2}=-\frac{g^{\prime 2} \cos ^{2} y \sin z}{g^{\prime \prime}}, \\
\mathfrak{U}_{3}=-\frac{g^{\prime 2} \sin y \cos y}{g^{\prime \prime}}, \mathfrak{U}_{4}=\frac{g^{\prime 2} \cos ^{2} y \cos z}{g^{\prime \prime}} ;
\end{array}\right\}
$$

and

$$
\left.\begin{array}{l}
\mathfrak{W}_{1}=0, \mathfrak{W}_{2}=\frac{x g^{\prime \prime} \cos z}{g^{\prime 2}-1}, \\
\mathfrak{W}_{3}=0, \mathfrak{W}_{4}=\frac{x g^{\prime \prime} \sin z}{g^{\prime 2}-1} .
\end{array}\right\}
$$

Thus, using (3.44)-(3.46) in (2.53), we obtain the components of the $\mathrm{LB}^{\mathrm{III}}$ operator of the rotational hypersurface $(3.1)$ as

$$
\left.\begin{array}{l}
\left(\Delta^{I I I} \Gamma\right)_{1}=\frac{\left(g^{\prime 2}-1\right)^{2}\left(-3 g^{\prime \prime 2}+g^{\prime} g^{\prime \prime \prime}\right)}{g^{\prime \prime 3}}, \\
\left(\Delta^{I I I} \Gamma\right)_{2}=\frac{\left(g^{\prime 2}-1\right)\left(g^{\prime}\left(1-g^{\prime 2}\right)\left(2 g^{\prime \prime 2}-g^{\prime} g^{\prime \prime \prime}\right)-2 x g^{\prime \prime 3}\right) \cos y \sin z}{g^{\prime 2} g^{\prime \prime 3}}, \\
\left(\Delta^{I I I} \Gamma\right)_{3}=-\frac{\left(g^{\prime 2}-1\right)\left(g^{\prime}\left(1-g^{\prime 2}\right)\left(2 g^{\prime \prime 2}-g^{\prime} g^{\prime \prime \prime}\right)-2 x g^{\prime \prime 3}\right) \sin y}{g^{\prime 2} g^{\prime \prime 3}}, \\
\left(\Delta^{I I I} \Gamma\right)_{4}=\frac{\left(g^{\prime 2}-1\right)\left(g^{\prime}\left(1-g^{\prime 2}\right)\left(2 g^{\prime \prime 2}-g^{\prime} g^{\prime \prime \prime}\right)-2 x g^{\prime \prime 3}\right) \cos y \cos z}{g^{\prime 2} g^{\prime \prime 3}},
\end{array}\right\}
$$

where $g(x) \neq a x+b, a, b \in \mathbb{R}$. So, from the proof of the Theorem 2.12, we can state the following theorem:

Theorem 3.12. The rotational hypersurface

$$
\Gamma(x, y, z)=\left(d_{4} \sqrt{2 x+\left(d_{4}\right)^{2}}+d_{5},-x \cos y \sin z,-x \sin y, x \cos y \cos z\right), d_{4}, d_{5} \in \mathbb{R}
$$

about timelike axis in $E_{1}^{4}$ is $L B^{I I I}$-minimal.

\section{Rotational hypersurfaces about lightlike axis in $E_{1}^{4}$}

In this section, we give some results for the Gaussian and mean curvatures of a rotational hypersurface with lightlike axis and this hypersurface's Gauss map. Also, we study the $\mathrm{LB}^{\mathrm{II}}$ and $\mathrm{LB}^{\mathrm{III}}$ operators on the rotational hypersurface with lightlike axis in $E_{1}^{4}$ and give some characterizations for $\mathrm{LB}^{\mathrm{II}}$-minimality and $\mathrm{LB}^{\mathrm{III}}$-minimality of this hypersurface.

\subsection{Curvatures of rotational hypersurfaces about lightlike axis in $E_{1}^{4}$}

For a differentiable function $h(x): I \subset \mathbb{R} \longrightarrow \mathbb{R}$, the rotational hypersurface which is obtained by rotating the profile curve $\gamma(x)=(x, h(x), 0,0)$ about lightlike axis $(1,1,0,0)$ is given by

$$
\begin{aligned}
\Gamma(x, y, z) & =\left[\begin{array}{cccc}
\frac{y^{2}+z^{2}}{2}+1 & -\frac{y^{2}+z^{2}}{2} & y & z \\
\frac{y^{2}+z^{2}}{2} & 1-\frac{y^{2}+z^{2}}{2} & y & z \\
y & -y & 1 & 0 \\
z & -z & 0 & 1
\end{array}\right] \cdot\left[\begin{array}{c}
x \\
h(x) \\
0 \\
0
\end{array}\right] \\
& =\left(\begin{array}{c}
\left(\frac{y^{2}+z^{2}}{2}+1\right) x-\frac{y^{2}+z^{2}}{2} h(x), \frac{y^{2}+z^{2}}{2} x+\left(1-\frac{y^{2}+z^{2}}{2}\right) h(x), \\
x y-h(x) y, x z-h(x) z
\end{array}\right),
\end{aligned}
$$

where $x \in \mathbb{R}-\{0\}$.

In the following figures, one can see the projections of the rotational hypersurface (4.1) for $h(x)=\sin x$ and $z=2$ into $x_{2} x_{3} x_{4}, x_{1} x_{3} x_{4}, x_{1} x_{2} x_{4}$ and $x_{1} x_{2} x_{3}$-spaces in (a), (b), (c) and (d), respectively. 


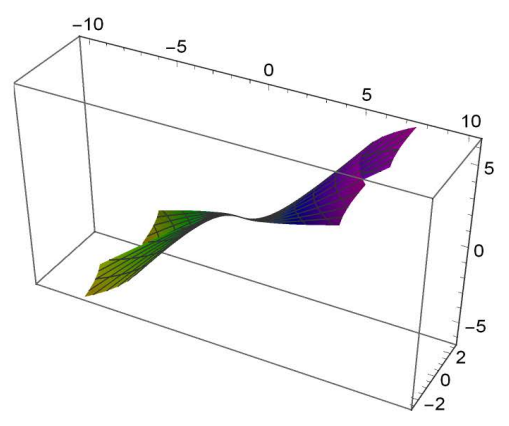

(a)

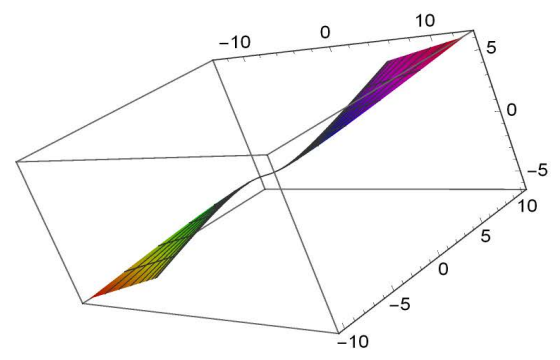

(c)

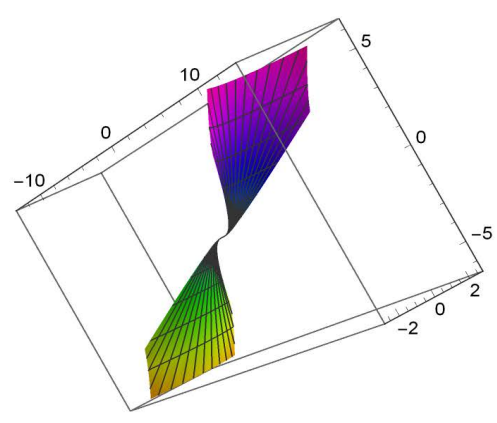

(b)

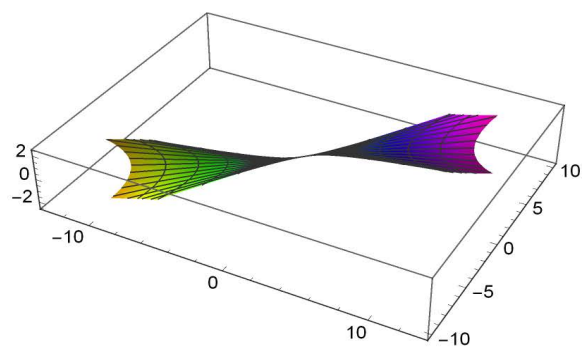

(d)

Figure 5

With the aid of the first differentials of (4.1) with respect to $x, y$ and $z$, the Gauss map of the rotational hypersurface (4.1) is obtained by

$$
N_{\Gamma}=\frac{\left(y^{2}+z^{2}-\left(y^{2}+z^{2}+2\right) h^{\prime}, y^{2}+z^{2}-2-\left(y^{2}+z^{2}\right) h^{\prime}, 2 y\left(1-h^{\prime}\right), 2 z\left(1-h^{\prime}\right)\right)}{2 \sqrt{1-h^{\prime 2}}}
$$

and from (4.2), we get

$$
\left\langle N_{\Gamma}, N_{\Gamma}\right\rangle=1
$$

Here, we state $h=h(x)$ and $h^{\prime}=\frac{d h(x)}{d x}$.

Also, from (1.5), we obtain the matrix of the first fundamental form, its inverse and determinant as

and

$$
\begin{gathered}
{\left[g_{i j}\right]=\left[\begin{array}{ccc}
h^{\prime 2}-1 & 0 & 0 \\
0 & (x-h)^{2} & 0 \\
0 & 0 & (x-h)^{2}
\end{array}\right],} \\
{\left[g^{i j}\right]=\left[\begin{array}{ccc}
\frac{1}{h^{\prime 2}-1} & 0 & 0 \\
0 & \frac{1}{(x-h)^{2}} & 0 \\
0 & 0 & \frac{1}{(x-h)^{2}}
\end{array}\right]}
\end{gathered}
$$

$$
\operatorname{det}\left[g_{i j}\right]=(x-h)^{4}\left(h^{\prime 2}-1\right),
$$

respectively. From (1.6), the matrix form of the second fundamental form of the hypersurface (4.1), its inverse and determinant as

$$
\begin{gathered}
{\left[h_{i j}\right]=\frac{1}{\sqrt{1-h^{\prime 2}}}\left[\begin{array}{ccc}
-h^{\prime \prime} & 0 & 0 \\
0 & (x-h)\left(h^{\prime}-1\right) & 0 \\
0 & 0 & (x-h)\left(h^{\prime}-1\right)
\end{array}\right],} \\
{\left[h^{i j}\right]=\sqrt{1-h^{\prime 2}}\left[\begin{array}{ccc}
-\frac{1}{h^{\prime \prime}} & 0 & 0 \\
0 & \frac{1}{(x-h)\left(h^{\prime}-1\right)} & 0 \\
0 & 0 & \frac{1}{(x-h)\left(h^{\prime}-1\right)}
\end{array}\right]}
\end{gathered}
$$


and

$$
\operatorname{det}\left[h_{i j}\right]=-\frac{(x-h)^{2} \sqrt{1-h^{\prime 2}} h^{\prime \prime}}{\left(1+h^{\prime}\right)^{2}},
$$

respectively and here, we state $h^{\prime \prime}=\frac{d^{2} h(x)}{d x^{2}}$. Hence, using (4.3), (4.6) and (4.9) in (1.8), we can give the following theorem:

Theorem 4.1. The Gaussian curvature of the rotational hypersurface (4.1) is

$$
K=\frac{h^{\prime \prime}}{(x-h)^{2}\left(h^{\prime}+1\right)^{2} \sqrt{1-h^{\prime 2}}} .
$$

From (4.10), we have

Corollary 4.2. The rotational hypersurface (4.1) is flat if and only if $h(x)=a x+b$, $a \in \mathbb{R}-\{-1,1\}, b \in \mathbb{R}$.

Also, using (4.5) and (4.7) in (1.7), the shape operator of the rotational hypersurface (4.1) is obtained by

$$
S=\frac{1}{\sqrt{1-h^{\prime 2}}}\left[\begin{array}{ccc}
\frac{h^{\prime \prime}}{\left(1-h^{\prime 2}\right)} & 0 & 0 \\
0 & \frac{h^{\prime}-1}{(x-h)} & 0 \\
0 & 0 & \frac{h^{\prime}-1}{(x-h)}
\end{array}\right] .
$$

So, from (1.9), (4.3) and (4.11), we get

Theorem 4.3. The mean curvature of the rotational hypersurface (4.1) is

$$
H=\frac{2\left(1-h^{\prime 2}\right)\left(-1+h^{\prime}\right)+(x-h) h^{\prime \prime}}{3(x-h)\left(1-h^{\prime 2}\right)^{3 / 2}} .
$$

If the rotational hypersurface (4.1) in $E_{1}^{4}$ is minimal, then from (4.12) it must be

$$
2\left(h^{\prime}-1\right)\left(1-h^{\prime 2}\right)+(x-h) h^{\prime \prime}=0 .
$$

Here, by taking $p(x)=h(x)-x$ in (4.13), we get

$$
2 p^{\prime 2}\left(p^{\prime}+2\right)+p p^{\prime \prime}=0 .
$$

If we take $p^{\prime}(x)=f(x)$, then we get

$$
p^{\prime \prime}=f^{\prime}=\frac{d f}{d p} f
$$

and by using (4.15) in (4.14), we have

$$
-\frac{d p}{p}=\frac{d f}{2 f(f+2)},
$$

where $p \neq 0 \neq f(f+2)$. By integrating (4.16), we get

$$
-\ln (p)=\frac{1}{4}\left(\ln \left(\frac{f}{2+f}\right)\right)-\ln (a),
$$

$a \in \mathbb{R}^{+}$and so

$$
f=\frac{2\left(\frac{a}{p}\right)^{4}}{1-\left(\frac{a}{p}\right)^{4}} .
$$

Since $f=p^{\prime}=\frac{d p}{d x}$, from (4.18) we have

$$
\frac{1-\left(\frac{a}{p}\right)^{4}}{2\left(\frac{a}{p}\right)^{4}} d p=d x
$$


and by integrating (4.19), we reach that

$$
p^{5}-5 a^{4} p=10 a^{4}(x+b),
$$

$b \in \mathbb{R}$. Finally using $p(x)=h(x)-x$ in $(4.20)$, we obtain the equation $(h-x)^{5}-5 a^{4} h=$ $5 a^{4}(x+2 b)$.

Conversely, if $h$ satisfies the equation $(h-x)^{5}-5 a^{4} h=5 a^{4}(x+2 b)$, then (4.12) vanishes. Thus, we can state the following theorem:

Theorem 4.4. The rotational hypersurface (4.1) in $E_{1}^{4}$ is minimal if and only if $h(x)$ satisfies the equation

$$
(h-x)^{5}-5 a^{4} h=5 a^{4}(x+2 b),
$$

$a \in \mathbb{R}^{+}, b \in \mathbb{R}$.

\subsection{Gauss map of the rotational hypersurface about lightlike axis in $E_{1}^{4}$}

From (4.2), let us parametrize the Gauss map of the rotational hypersurface about lightlike axis in $E_{1}^{4}$ as

$\Gamma_{G}(x, y, z)=\frac{\left(y^{2}+z^{2}-\left(y^{2}+z^{2}+2\right) h^{\prime}, y^{2}+z^{2}-2-\left(y^{2}+z^{2}\right) h^{\prime}, 2 y\left(1-h^{\prime}\right), 2 z\left(1-h^{\prime}\right)\right)}{2 \sqrt{1-h^{\prime 2}}}$.

Then, the normal of (4.22) is

$$
N_{G}=\frac{\left(-y^{2}-z^{2}+\left(y^{2}+z^{2}+2\right) h^{\prime},-y^{2}-z^{2}+2+\left(y^{2}+z^{2}\right) h^{\prime}, 2 y\left(h^{\prime}-1\right), 2 z\left(h^{\prime}-1\right)\right)}{2 \sqrt{1-h^{\prime 2}}}
$$

and from (4.23), we have

$$
\left\langle N_{G}, N_{G}\right\rangle=1
$$

From (1.5), we obtain the matrix of the first fundamental form, its inverse and determinant as

$$
\begin{aligned}
{\left[g_{i j}\right]_{G}=} & {\left[\begin{array}{ccc}
-\frac{h^{\prime \prime 2}}{\left(h^{\prime 2}-1\right)^{2}} & 0 & 0 \\
0 & \frac{1-h^{\prime}}{1+h^{\prime}} & 0 \\
0 & 0 & \frac{1-h^{\prime}}{1+h^{\prime}}
\end{array}\right], } \\
{\left[g^{i j}\right]_{G}=} & {\left[\begin{array}{ccc}
-\frac{\left(h^{\prime 2}-1\right)^{2}}{h^{\prime \prime 2}} & 0 & 0 \\
0 & \frac{1+h^{\prime}}{1-h^{\prime}} & 0 \\
0 & 0 & \frac{1+h^{\prime}}{1-h^{\prime}}
\end{array}\right] }
\end{aligned}
$$

and

$$
\operatorname{det}\left(\left[g_{i j}\right]_{G}\right)=-\frac{h^{\prime \prime 2}}{\left(h^{\prime}+1\right)^{4}}
$$

respectively. From (1.6), the matrix form of the second fundamental form of (4.22), its inverse and determinant as

$$
\begin{gathered}
{\left[h_{i j}\right]_{G}=-\left[\begin{array}{ccc}
\frac{h^{\prime 2}}{\left(h^{\prime 2}-1\right)^{2}} & 0 & 0 \\
0 & \frac{-1+h^{\prime}}{1+h^{\prime}} & 0 \\
0 & 0 & \frac{-1+h^{\prime}}{1+h^{\prime}}
\end{array}\right],} \\
{\left[h^{i j}\right]_{G}=\left[\begin{array}{ccc}
-\frac{\left(h^{\prime 2}-1\right)^{2}}{h^{\prime \prime 2}} & 0 & 0 \\
0 & \frac{1+h^{\prime}}{1-h^{\prime}} & 0 \\
0 & 0 & \frac{1+h^{\prime}}{1-h^{\prime}}
\end{array}\right]}
\end{gathered}
$$

and

$$
\operatorname{det}\left(\left[h_{i j}\right]_{G}\right)=-\frac{h^{\prime \prime 2}}{\left(h^{\prime}+1\right)^{4}}
$$


respectively. Hence, using (4.24), (4.27) and (4.30) in (1.8), we can give the following theorem:

Theorem 4.5. The Gaussian curvature of (4.22) is

$$
K_{G}=1 .
$$

Also, using (4.26) and (4.28) in (1.7), the shape operator of (4.22) is obtained by

$$
S_{G}=\left[\begin{array}{lll}
1 & 0 & 0 \\
0 & 1 & 0 \\
0 & 0 & 1
\end{array}\right] .
$$

So, from (1.9), (4.24) and (4.32), we get

Theorem 4.6. The mean curvature of (4.22) is

$$
H_{G}=1 .
$$

\subsection{The second Laplace-Beltrami operator on rotational hypersurface about lightlike axis in $E_{1}^{4}$}

Using the same procedure in subsection 2.3, we obtain the components $U_{i}, V_{i}$ and $W_{i}$ which are defined in $(2.42)$ as

$$
\left.\begin{array}{c}
U_{1}=\frac{(x-h)\left(h^{\prime}-1\right)\left(y^{2}+z^{2}+2-\left(y^{2}+z^{2}\right) h^{\prime}\right)}{2 \sqrt{-h^{\prime \prime} \sqrt{1-h^{\prime 2}}}}, U_{2}=\frac{(h-x)\left(h^{\prime}-1\right)\left(\left(y^{2}+z^{2}-2\right) h^{\prime}-y^{2}-z^{2}\right)}{2 \sqrt{-h^{\prime \prime} \sqrt{1-h^{\prime 2}}}}, \\
\left.\begin{array}{c}
U_{3}=\frac{y(h-x)\left(1-h^{\prime}\right)^{2}}{\sqrt{-h^{\prime \prime} \sqrt{1-h^{\prime 2}}}}, U_{4}=\frac{z(h-x)\left(1-h^{\prime}\right)^{2}}{\sqrt{-h^{\prime \prime} \sqrt{1-h^{\prime 2}}}} ; \\
V_{1}=\frac{y(h-x) h^{\prime \prime}}{\sqrt{-h^{\prime \prime} \sqrt{1-h^{\prime 2}}}}, V_{2}=\frac{y(h-x) h^{\prime \prime}}{\sqrt{-h^{\prime \prime} \sqrt{1-h^{\prime 2}}}}, \\
V_{3}=\frac{\left(h-x h^{\prime \prime}\right.}{\sqrt{-h^{\prime \prime} \sqrt{1-h^{\prime 2}}}}, V_{4}=0
\end{array}\right\}
\end{array}\right\}
$$

and

$$
\left.\begin{array}{l}
W_{1}=-\frac{z(x-h) h^{\prime \prime}}{\sqrt{-h^{\prime \prime} \sqrt{1-h^{\prime 2}}}}, W_{2}=-\frac{z(x-h) h^{\prime \prime}}{\sqrt{-h^{\prime \prime} \sqrt{1-h^{\prime 2}}}}, \\
W_{3}=0, W_{4}=-\frac{(x-h) h^{\prime \prime}}{\sqrt{-h^{\prime \prime} \sqrt{1-h^{\prime 2}}}} .
\end{array}\right\}
$$

Thus, using (4.34)-(4.36) in (2.41), we obtain the components of the $\mathrm{LB}^{\mathrm{II}}$ operator of the rotational hypersurface (4.1) as

$$
\begin{gathered}
-2\left(h^{\prime}-1\right)^{2}\left(h^{\prime}+1\right)\left(\left(y^{2}+z^{2}\right) h^{\prime}-y^{2}-z^{2}-2\right) h^{\prime \prime} \\
\left(\Delta^{I I} \Gamma\right)_{1}=-\frac{+(x-h)\left(-4\left(y^{2}+z^{2}-1\right)+\left(y^{2}+z^{2}+6\right) h^{\prime}+3\left(y^{2}+z^{2}\right) h^{\prime 2}\right) h^{\prime \prime 2}}{-(x-h)\left(\left(y^{2}+z^{2}\right) h^{\prime}-y^{2}-z^{2}-2\right)\left(h^{\prime 2}-1\right) h^{\prime \prime \prime}} \\
4(x-h) \sqrt{1-h^{\prime 2}} h^{\prime \prime 2} \\
\left(\Delta^{I I} \Gamma\right)_{2}=-\frac{-2\left(h^{\prime}-1\right)^{2}\left(h^{\prime}+1\right)\left(\left(y^{2}+z^{2}-2\right) h^{\prime}-y^{2}-z^{2}\right) h^{\prime \prime}}{+(x-h)\left(-4\left(y^{2}+z^{2}-3\right)+\left(y^{2}+z^{2}+4\right) h^{\prime}+3\left(y^{2}+z^{2}-2\right) h^{\prime 2}\right) h^{\prime \prime 2}} \\
\left(\Delta^{I I} \Gamma\right)_{3}=\frac{y\left(-1+h^{\prime}\right)\left(2\left(h^{\prime}-1\right)^{2}\left(h^{\prime}+1\right) h^{\prime \prime}-(x-h)\left(3 h^{\prime}+4\right) h^{\prime \prime 2}+(x-h)\left(h^{\prime 2}-1\right) h^{\prime \prime \prime}\right)}{2(x-h) \sqrt{1-h^{\prime 2}} h^{\prime \prime 2}}, \\
\left(\Delta^{I I} \Gamma\right)_{4}=\frac{z\left(-1+h^{\prime}\right)\left(2\left(h^{\prime}-1\right)^{2}\left(h^{\prime}+1\right) h^{\prime \prime}-(x-h)\left(3 h^{\prime}+4\right) h^{\prime \prime 2}+(x-h)\left(h^{\prime 2}-1\right) h^{\prime \prime \prime}\right)}{2(x-h) \sqrt{1-h^{\prime 2}} h^{\prime \prime 2}},
\end{gathered}
$$

where $h(x) \neq a x+b, a, b \in \mathbb{R}$ and $h^{\prime \prime \prime}=\frac{d^{3} h(x)}{d x^{3}}$. 


\subsection{The third Laplace-Beltrami operator on rotational hypersurface about lightlike axis in $E_{1}^{4}$}

The matrix of third fundamental form, its inverse and the determinant are obtained by

$$
\begin{gathered}
{\left[m_{i j}\right]=\left[\begin{array}{ccc}
-\frac{h^{\prime 2}}{\left(h^{\prime 2}-1\right)^{2}} & 0 & 0 \\
0 & \frac{1-h^{\prime}}{1+h^{\prime}} & 0 \\
0 & 0 & \frac{1-h^{\prime}}{1+h^{\prime}}
\end{array}\right],} \\
{\left[m^{i j}\right]=\left[\begin{array}{ccc}
-\frac{\left(h^{\prime 2}-1\right)^{2}}{h^{\prime \prime 2}} & 0 & 0 \\
0 & \frac{1+h^{\prime}}{1-h^{\prime}} & 0 \\
0 & 0 & \frac{1+h^{\prime}}{1-h^{\prime}}
\end{array}\right]}
\end{gathered}
$$

and

$$
\operatorname{det}\left[m_{i j}\right]=-\frac{h^{\prime \prime 2}}{\left(h^{\prime}+1\right)^{4}},
$$

respectively. Using the same procedure in subsection 2.4, we obtain the components $\mathfrak{U}_{i}$, $\mathfrak{V}_{i}$ and $\mathfrak{W}_{i}$ which are defined in (2.54) as

$$
\left.\begin{array}{c}
\mathfrak{U}_{1}=\frac{\left(1-h^{\prime}\right)^{2}\left(y^{2}+z^{2}+2-\left(y^{2}+z^{2}\right) h^{\prime}\right)}{2 h^{\prime \prime}}, \mathfrak{U}_{2}=\frac{\left(1-h^{\prime}\right)^{2}\left(y^{2}+z^{2}-\left(y^{2}+z^{2}-2\right) h^{\prime}\right)}{2 h^{\prime \prime}}, \\
\mathfrak{U}_{3}=\frac{y\left(1-h^{\prime}\right)^{3}}{h^{\prime \prime}}, \mathfrak{U}_{4}=\frac{z\left(1-h^{\prime}\right)^{3}}{h^{\prime \prime}} ; \\
\mathfrak{V}_{1}=\frac{y(x-h) h^{\prime \prime}}{h^{\prime 2}-1}, \mathfrak{V}_{2}=\frac{y(x-h) h^{\prime \prime}}{h^{\prime 2}-1}, \\
\mathfrak{V}_{3}=\frac{(x-h) h^{\prime \prime}}{h^{\prime 2}-1}, \mathfrak{V}_{4}=0
\end{array}\right\}
$$

and

$$
\left.\begin{array}{l}
\mathfrak{W}_{1}=\frac{z(x-h) h^{\prime \prime}}{h^{\prime 2}-1}, \mathfrak{W}_{2}=\frac{z(x-h) h^{\prime \prime}}{h^{\prime 2}-1}, \\
\mathfrak{W}_{3}=0, \mathfrak{W}_{4}=\frac{(x-h) h^{\prime \prime}}{h^{\prime 2}-1} .
\end{array}\right\}
$$

Thus, using (4.41)-(4.43) in (2.53), we obtain the components of the $\mathrm{LB}^{\mathrm{III}}$ operator of the rotational hypersurface (4.1) as

$$
\begin{aligned}
\left(1+h^{\prime}\right)^{2}\left(-\left(h^{\prime}-1\right)^{2}\left(h^{\prime}+1\right)\left(3\left(y^{2}+z^{2}\right) h^{\prime}-3 y^{2}-3 z^{2}-4\right) h^{\prime \prime 2}\right. \\
\left(\Delta^{I I I} \Gamma\right)_{1}=-\frac{\left.+4(x-h) h^{\prime \prime 3}+\left(h^{\prime}-1\right)^{3}\left(h^{\prime}+1\right)\left(\left(y^{2}+z^{2}\right) h^{\prime}-y^{2}-z^{2}-2\right) h^{\prime \prime \prime}\right)}{2\left(h^{\prime 2}-1\right) h^{\prime \prime 3}} \\
\left(\Delta^{I I I} \Gamma\right)_{2}=-\frac{\left.+4(x-h) h^{\prime \prime 3}+\left(h^{\prime}-1\right)^{3}\left(h^{\prime}+1\right)\left(\left(y^{2}+z^{2}-2\right) h^{\prime}-y^{2}-z^{2}\right) h^{\prime \prime \prime}\right)}{2\left(h^{\prime 2}-1\right) h^{\prime \prime 3}} \\
\left(\Delta^{I I I} \Gamma\right)_{3}=-\frac{y\left(h^{\prime 2}-1\right)^{2}\left(-3 h^{\prime \prime 2}+\left(h^{\prime}-1\right) h^{\prime \prime \prime}\right)}{h^{\prime \prime 3}}, \\
\left(\Delta^{I I I} \Gamma\right)_{4}=-\frac{z\left(h^{\prime 2}-1\right)^{2}\left(-3 h^{\prime \prime 2}+\left(h^{\prime}-1\right) h^{\prime \prime \prime}\right)}{h^{\prime \prime 3}}
\end{aligned}
$$

where $h(x) \neq a x+b, a, b \in \mathbb{R}$. So,

Theorem 4.7. The rotational hypersurface

$$
\Gamma(x, y, z)=\left(\begin{array}{c}
\left(\frac{y^{2}+z^{2}}{2}+1\right) x-\frac{y^{2}+z^{2}}{2}\left(\frac{2 x d_{6}-2 \sqrt{-2 d_{6}\left(x+d_{7}\right)}-1}{2 d_{6}}\right), \\
\frac{y^{2}+z^{2}}{2} x+\left(1-\frac{y^{2}+z^{2}}{2}\right)\left(\frac{2 x d_{6}-2 \sqrt{-2 d_{6}\left(x+d_{7}\right)}-1}{2 d_{6}}\right), \\
x y-\left(\frac{2 x d_{6}-2 \sqrt{-2 d_{6}\left(x+d_{7}\right)}-1}{2 d_{6}}\right) y, x z-\left(\frac{2 x d_{6}-2 \sqrt{-2 d_{6}\left(x+d_{7}\right)}-1}{2 d_{6}}\right) z
\end{array}\right), d_{6}, d_{7} \in \mathbb{R}
$$

about lightlike axis in $E_{1}^{4}$ is $L B^{I I I}$-minimal.

Proof. The solution of the differential equation

$$
-3 h^{\prime \prime 2}+\left(h^{\prime}-1\right) h^{\prime \prime \prime}=0
$$


is obtained as

$$
h(x)=\frac{x d_{6}-\sqrt{-2 d_{6}\left(x+d_{7}\right)}}{d_{6}}+d_{8}, \quad d_{6}, d_{7}, d_{8} \in \mathbb{R} .
$$

If we use this function in the first two components of $\mathrm{LB}^{\mathrm{III}}$ operator $\Delta^{I I I} \Gamma$, then for these components to be zero, it must be $d_{8}=\frac{-1}{2 d_{6}}$. So, the proof completes.

Here, we must note that the Gaussian and mean curvatures, which are given for the rotational hypersurfaces in this study, can be obtained by taking $a=b=0$ in [11].

Acknowledgment. The authors gratefully thank to the Referees for the constructive comments and recommendations which definitely help to improve the readability and quality of the paper.

\section{References}

[1] M. Altın, A. Kazan and H.B. Karadăg, Monge Hypersurfaces in Euclidean 4-Space with Density, Journal of Polytechnic, 23 (1), 207-214, 2020.

[2] K. Arslan, B.K. Bayram, B. Bulca and G. Öztürk, Generalized Rotation Surfaces in $E^{4}$, Results. Math. 61, 315-327, 2012.

[3] K. Arslan, B.K. Bayram, B. Bulca and G. Öztürk, On translation surfaces in 4dimensional Euclidean space, Acta et Com. Uni. Tar. De Math. 20 (2), 123-133, 2016.

[4] A. Arvanitoyeorgos, G. Kaimakamis and M. Magid, Lorentz Hypersurfaces in $E_{1}^{4}$ satisfying $\Delta \vec{H}=\alpha \vec{H}$, Illinois J. Math. 53 (2), 581-590, 2009.

[5] M. Bekkar and B. Senoussi, Translation surfaces in the 3-dimensional space satisfying $\Delta^{I I I} r_{i}=\mu_{i} r_{i}$, J. Geom. 103, 367-374, 2012.

[6] B.Y. Chen and S. Ishikawa, On classification of some surfaces of revolution of finite type, Tsukuba J. Math. 17 (1), 287-298, 1993.

[7] Q-M. Cheng and Q-R. Wan, Complete Hypersurfaces of $R^{4}$ with Constant Mean Curvature, Monatsh. Math. 118, 171-204, 1994.

[8] A. Çakmak, M.K. Karacan, S. Kızıltuğ and D.W. Yoon, Translation Surfaces in the 3-Dimensional Galilean Space Satisfying $\Delta^{I I} x_{i}=\lambda_{i} x_{i}$, Bull. Korean Math. Soc. 54 (4), 1241-1254, 2017.

[9] U. Dursun, Rotational Hypersurfaces in Lorentz-Minkowski Space with Constant Mean Curvature, Taiwanese J. Math. 14 (2), 685-705, 2010.

[10] G. Ganchev and V. Milousheva, General rotational surfaces in the 4-dimensional Minkowski space, Turkish J. Math. 38, 883-895, 2014.

[11] E. Güler, Helical Hypersurfaces in Minkowski Geometry $E_{1}^{4}$, Symmetry, 12, 1206, 2020.

[12] E. Güler and A.G. Karaalp, Dini Type Helicoidal Hypersurface in 4-Space, Ikonian Journal of Mathematics 1 (1), 26-34, 2019.

[13] E. Güler and Ö. Kişi, Dini-type helicoidal hypersurfaces with timelike axis in Minkowski 4-space $E_{1}^{4}$, Mathematics, 7 (205), 1-8, 2019.

[14] E. Güler, H.H. Hacisalihoğlu and Y.H. Kim, The Gauss map and the third LaplaceBeltrami operator of the rotational hypersurface in 4-Space, Symmetry, 10 (9), 1-11, 2018.

[15] T.H. Hasanis and T.H. Vlachos, Hypersurfaces in $E^{4}$ with Harmonic Mean Curvature Vector Field, Math. Nachr. 172, 145-169, 1995.

[16] S. Izumiya, M.D.C.R. Fuster and K. Saji, Flat Lightlike Hypersurfaces in LorentzMinkowski 4-Space, J. Geom. Phys. 59, 1528-1546, 2009.

[17] C. Moore, Surfaces of rotation in a space of four dimensions, Ann. Math. 21, 81-93, 1919. 
[18] M. Moruz and M.I. Munteanu, Minimal translation hypersurfaces in $E^{4}$, J. Math. Anal. Appl. 439 (2), 798-812, 2016.

[19] D.W. Yoon, Rotation surfaces with finite type Gauss map in $E^{4}$, Indian J. Pure Appl. Math. 32 (12), 1803-1808, 2001. 